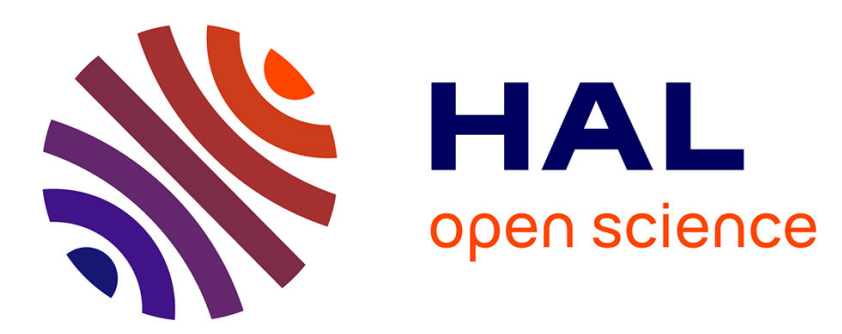

\title{
Fast Relaxation Solvers for Hyperbolic-Elliptic Phase Transition Problems
}

Christophe Chalons, Frédéric Coquel, Patrick Engel, Christian Rohde

\section{To cite this version:}

Christophe Chalons, Frédéric Coquel, Patrick Engel, Christian Rohde. Fast Relaxation Solvers for Hyperbolic-Elliptic Phase Transition Problems. SIAM Journal on Scientific Computing, 2012, 34 (3), http://dx.doi.org/10.1137/110848815. hal-00726479

\section{HAL Id: hal-00726479 \\ https://hal.science/hal-00726479}

Submitted on 30 Aug 2012

HAL is a multi-disciplinary open access archive for the deposit and dissemination of scientific research documents, whether they are published or not. The documents may come from teaching and research institutions in France or abroad, or from public or private research centers.
L'archive ouverte pluridisciplinaire HAL, est destinée au dépôt et à la diffusion de documents scientifiques de niveau recherche, publiés ou non, émanant des établissements d'enseignement et de recherche français ou étrangers, des laboratoires publics ou privés. 


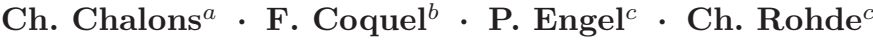 \\ Fast Relaxation Solvers for Hyperbolic-Elliptic Phase Transition Problems
}

\author{
Stuttgart, September 2011 \\ ${ }^{a}$ Université Paris Diderot Paris 7 \& Laboratoire Jacques-Louis Lions \\ U.M.R. 7598, Boîte courrier 187, 75252 Paris Cedex 05, France \\ chalons@math.jussieu.fr \\ ${ }^{b}$ CNRS \& Centre de Mathématiques Appliquées U.M.R 7641 \\ Ecole Polytechnique, Route de Saclay, 91128 Palaiseau Cedex, France \\ frederic.coquel@cmap.polytechnique.fr \\ ${ }^{c}$ Institute of Applied Analysis and Numerical Simulation, University of Stuttgart, \\ Pfaffenwaldring 57, 70569 Stuttgart/ Germany \\ patrick.engel, christian.rohde@mathematik.uni-stuttgart.de \\ www.ians.uni-stuttgart.de
}

\begin{abstract}
Phase transition problems in compressible media can be modelled by mixed hyperbolicelliptic systems of conservation laws. Within this approach phase boundaries are understood as shock waves that satisfy additional constraints, sometimes called kinetic relations. In recent years several tracking-type algorithms have been suggested for numerical approximation. Typically a core piece of these algorithms is the usage of exact Riemann solvers incorporating the kinetic relation at the location of phase boundaries. However, exact Riemann solvers are computationally expensive or even not available.

In this paper we present a class of approximate Riemann solvers for hyperbolic-elliptic models that relies on a generalized relaxation procedure. It preserves in particular the kinetic relation for phase boundaries exactly and gives for isolated phase transitions the correct solutions. In combination with a novel sub-iteration procedure the approximate Riemann solvers are used in the tracking algorithms. The efficiency of the approach is validated on a barotropic system with linear kinetic relation where exact Riemann solvers are available. For a nonlinear kinetic relation and a thermoelastic system we use the new method to gain information on the Riemann problem. Up to our knowledge an exact solution for arbitrary Riemann data is currently not available in these cases.
\end{abstract}

Preprint Series

Issue No. 2011

Stuttgart Research Centre for Simulation Technology (SRC SimTech)

SimTech - Cluster of Excellence

Pfaffenwaldring $7 \mathrm{a}$

70569 Stuttgart

publications@simtech.uni-stuttgart.de

www.simtech.uni-stuttgart.de 


\title{
Fast Relaxation Solvers for Hyperbolic-Elliptic Phase Transition Problems
}

\author{
Ch. Chalons* \\ F. Coquel ${ }^{\dagger}$ \\ P. Engel $l^{\ddagger}$ \\ Ch. Rohde
}

September 21, 2011

\begin{abstract}
Phase transition problems in compressible media can be modelled by mixed hyperbolicelliptic systems of conservation laws. Within this approach phase boundaries are understood as shock waves that satisfy additional constraints, sometimes called kinetic relations. In recent years several tracking-type algorithms have been suggested for numerical approximation. Typically a core piece of these algorithms is the usage of exact Riemann solvers incorporating the kinetic relation at the location of phase boundaries. However, exact Riemann solvers are computationally expensive or even not available.

In this paper we present a class of approximate Riemann solvers for hyperbolic-elliptic models that relies on a generalized relaxation procedure. It preserves in particular the kinetic relation for phase boundaries exactly and gives for isolated phase transitions the correct solutions. In combination with a novel sub-iteration procedure the approximate Riemann solvers are used in the tracking algorithms.

The efficiency of the approach is validated on a barotropic system with linear kinetic relation where exact Riemann solvers are available. For a nonlinear kinetic relation and a thermoelastic system we use the new method to gain information on the Riemann problem. Up to our knowledge an exact solution for arbitrary Riemann data is currently not available in these cases.
\end{abstract}

\section{Introduction}

Mixed hyperbolic-elliptic systems of conservation laws are by now well-accepted models to describe the inviscid dynamics of phase transitions in compressible media. As an example let us mention the equations of ideal hydrodynamics with a van-der-Waals type pressure law to describe the compressible motion of a homogeneous fluid in liquid and vapor phases [27]. Also the evolution of solid-solid transformations in elastic materials is a typical instance [1].

In one space dimension these models take the form

$$
U_{t}+F(U)_{x}=0 \text { in } \mathbb{R} \times(0, \infty) .
$$

*Université Paris Diderot Paris 7 \& Laboratoire Jacques-Louis Lions U.M.R. 7598, Boîte courrier 187, 75252 Paris Cedex 05, France.

chalons@math.jussieu.fr

${ }^{\dagger}$ CNRS \& Centre de Mathématiques Appliquées U.M.R 7641

Ecole Polytechnique, Route de Saclay, 91128 Palaiseau Cedex, France.

frederic.coquel@cmap.polytechnique.fr

${ }^{\ddagger}$ Institut für Angewandte Analysis und Numerische Simulation, University of Stuttgart, Pfaffenwaldring 57, D-70569 Stuttgart, Germany.

\{patrick.engel,christian.rohde\}@mathematik.uni-stuttgart.de 
For an open convex set $\mathcal{U} \subset \mathbb{R}^{m}$ the function $F \in C^{1}\left(\mathcal{U}, \mathbb{R}^{m}\right)$ is the given flux and $m \geq 1$ is the number of components for the unknown $U=U(x, t)$. We assume that the set $\mathcal{U}$ is decomposed into the convex open sets $\mathcal{U}_{1}, \mathcal{U}_{2} \subset \mathcal{U}$ and the open set $\mathcal{U}_{\mathrm{e}} \subset \mathcal{U}$ with pairwise empty intersection such that

$$
\overline{\mathcal{U}}=\overline{\mathcal{U}_{1} \cup \mathcal{U}_{2} \cup \mathcal{U}_{\mathrm{e}}}, \quad \overline{\mathcal{U}_{1}} \cap \overline{\mathcal{U}_{2}}=\emptyset
$$

with

$$
\begin{gathered}
D F(U) \text { is diagonalizable in } \mathbb{R} \text { for } U \in \mathcal{U}_{1} \cup \mathcal{U}_{2}, \\
\operatorname{spec}(D F(U)) \cap(\mathbb{C} \backslash \mathbb{R}) \neq \emptyset \text { for } U \in \mathcal{U}_{\mathrm{e}} .
\end{gathered}
$$

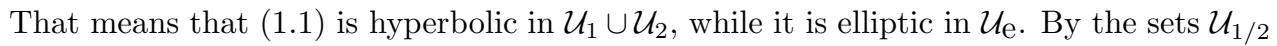
two different phases are characterized.

We will focus on the initial value problem, i.e., given a function $U_{0}: \mathbb{R} \rightarrow \mathcal{U}_{1} \cup \mathcal{U}_{2}$ we consider

$$
U(\cdot, 0)=U_{0} \text { in } \mathbb{R}
$$

and finally search for the unknown $U: \mathbb{R} \times[0, \infty) \rightarrow \mathcal{U}_{1} \cup \mathcal{U}_{2}$ avoiding values in $\mathcal{U}_{\mathrm{e}}$.

An important choice of initial datum is the Riemann problem with states in two different phases, e.g.

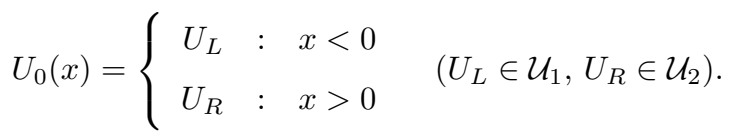

For many cases exact (entropy-consistent) Riemann solutions are known which consist of standard waves as rarefaction waves or Laxian shock waves satisfying the RankineHugoniot conditions. Phase boundaries appear as usually non-Laxian shock waves. To select a unique weak solution of the Riemann problem requires typically to enforce an additional condition ${ }^{1}$ on the traces at the phase boundary. Denoting the trace states by $U_{ \pm}$with e.g. $U_{-} \in \mathcal{U}_{1}$ and $U_{+} \in \mathcal{U}_{2}$ and given some function $\mathcal{K}: \mathcal{U}_{1} \times \mathcal{U}_{2} \rightarrow \mathbb{R}$ the so-called kinetic relation writes in the form

$$
\mathcal{K}\left(U_{-}, U_{+}\right)=0 .
$$

We refer for a general theory on Riemann solutions to [18] and for specific examples to $[14,20,21,23]$ without making any attempt to cite the complete literature on the topic. For the numerical solution of (1.1) with arbitrary initial data in $\mathcal{U}_{1} \cup \mathcal{U}_{2}$ standard finitevolume schemes cannot be used as they smear out discontinuities and thus produce spurious values in $\mathcal{U}_{\mathrm{e}}$ at the phase boundary. Therefore tracking type algorithms have been suggested which resolve the phase boundary over one cell boundary [6, 23, 28]. These methods usually require the exact solution of Riemann problems at the phase boundaries. In Section 2 we present a sample algorithm of this class. Let us note here that the exact Riemann solution is also used in multidimensional calculations to compute fluxes in normal direction of mesh edges [15].

It turns out that the computation of exact solutions dominates the total computational effort of the schemes. Therefore it is mandatory to develop efficient approximate solutions. We suggest in this paper an approach that relies on a relaxation approximation of (1.1) of type

$$
\tilde{U}_{t}^{\lambda}+\tilde{F}\left(\tilde{U}^{\lambda}\right)_{x}=\lambda R\left(\tilde{U}^{\lambda}\right) \text { in } \mathbb{R} \times(0, \infty),
$$

with $\lambda$ some positive relaxation parameter. Here the unknown $\tilde{U}^{\lambda}$ belongs to a phase space $\tilde{\mathcal{U}} \subset \mathbb{R}^{n}$ with $n>m$. The system (1.6) is hyperbolic in $\tilde{\mathcal{U}}$ and is constructed in such a way that its characteristic fields are linearly degenerate in $\tilde{\mathcal{U}}$. Thus the Riemann problem for (the homogeneous part of) (1.6) can be easily solved. For problems with simply connected hyperbolic state space relaxation approximations as (1.6) are widely

\footnotetext{
${ }^{1}$ We assume the setting, where supplementing one condition suffices.
} 
used $[9,13,17]$. As we are interested in two-phase solutions we replace at the phase boundary one of the Rankine-Hugoniot relations for (1.6) by the kinetic relation (1.5). This new approach is presented and analyzed in detail for the example of a barotropic material in Section 3. Using the resulting approximate Riemann solver in the tracking algorithm leads to a robust scheme. However it is computationally not much more efficient as the version with an exact solver. By efficiency we mean run-time needed to stay below a prescribed error tolerance. We increase now computational efficiency essentially by coupling the scheme with sub-iteration procedures. The iteration scheme has its own interest independently of mixed-type system as it provides an approximation of the exact Riemann solution for (1.1) by a sequence of relaxation approximations built from (1.6). The iteration techniques presented are even more general. They can be applied to improve e.g. the resolution of contact waves in gas dynamics and are not restricted to the field of non-classical waves.

In Section 4 we address the study of a non-barotropic material. In this case - up to our knowledge - complete exact Riemann solutions have not been found up to now (cf. [2, 16] for work in this direction). We apply the method developed in Section 3 to the setting of thermoelastic materials. In this way one can get highly accurate informations on e.g. the Riemann problem. We view this part of our work not merely as a numerical contribution but as a step to understand the dynamics of non-barotropic materials.

\section{$2 \quad$ Tracking-Type Algorithms}

Stable numerical schemes to approximate weak solutions of the initial value problem (1.1), (1.3) that involve nonclassical shock waves have been developed over the last two decades. We refer to $[4,6,10,11,7,12,19,22,23,25,28]$ to mention just a few works. Here we review shortly a basic version of a typical tracking-type method. As we will see below the algorithm needs as its core tool an exact Riemann solver for states $U_{L}, U_{R}$ in two different phases.

Let us assume for the moment that a unique exact (entropy-consistent) Riemann solution for $(1.1),(1.4)$ is available, which contains a single phase transition connecting states $U_{-}$ and $U_{+}$with speed $\sigma \in \mathbb{R}$ such that the Rankine-Hugoniot relations and a given kinetic relation (1.5) are satisfied.

We consider a bounded initial function $U_{0}: \mathbb{R} \rightarrow \mathcal{U}_{1} \cup \mathcal{U}_{2}$ with $K \in \mathbb{N}$ points of discontinuity which are supposed to be located at $\Gamma_{k}^{0} \in \mathbb{R}$ for $k \in \mathbf{K}^{0}:=\{1, \ldots, K\}$ and constitute a phase boundary. We assume that $\mathbb{R}$ is partitioned into cells $\left\{T_{i}\right\}_{i \in \mathbb{Z}}$ with $T_{i}=\left[x_{i-1 / 2}, x_{i+1 / 2}\right)$ such that for any $k \in \mathbf{K}^{0}$ there is a unique index $j=j(0, k) \in \mathbb{Z}$ with $\Gamma_{k}^{0}=x_{j-1 / 2}$.

In the initial step we define the cell averages

$$
U_{i}^{0}=\frac{1}{\Delta x_{i}} \int_{T_{i}} U_{0}(x) d x, \quad \Delta x_{i}=x_{i+1 / 2}-x_{i-1 / 2}, \quad(i \in \mathbb{Z}) .
$$

By construction and convexity of $\mathcal{U}_{1}, \mathcal{U}_{2}$ we have $U_{i}^{0} \in \mathcal{U}_{1} \cup \mathcal{U}_{2}$. Furthermore the discrete data exhibits phase changes only across edge points $x_{j(0, k)-1 / 2}, k \in \mathbf{K}^{0}$.

The sample algorithm to compute the $(n+1)$-th time level $t^{n+1}$, the phase boundary locations $\left\{\Gamma_{k}^{n+1}\right\}_{k \in \mathbf{K}^{n+1}}$ and the average data $\left\{U_{i}^{n+1}\right\}_{i \in \mathbb{Z}}$ from $\left\{\Gamma_{k}^{n}\right\}_{k \in \mathbf{K}^{n}}$ and $\left\{U_{i}^{n}\right\}_{i \in \mathbb{Z}}$ at time $t^{n}$ now reads as follows.

Algorithm 2.1 Let $n \in \mathbb{N}_{0}$.

1. Denote for any $k \in \mathbf{K}^{n}$ the smallest index $j=j(n, k) \in \mathbb{Z}$ with $\left|\Gamma_{k}^{n}-x_{j-1 / 2}\right|=$ $\min _{l \in \mathbb{Z}}\left\{\left|\Gamma_{k}^{n}-x_{l-1 / 2}\right|\right\}$. For $k \in \mathbf{K}^{n}$ compute the exact Riemann solution of (1.1) with

$$
U_{L}=U_{j-1}^{n}, \quad U_{R}=U_{j}^{n} .
$$

Denote the end states of the phase boundary by $U_{-}^{n, k}, U_{+}^{n, k}$ and its speed by $\sigma_{n, k}$. 
2. Let $\Delta t^{n}>0$ be chosen such that

$$
\Delta t^{n} \max \left\{\sup _{i \in \mathbb{Z}}\left\{\bar{\lambda}\left(U_{i}^{n}\right)\right\}, \sigma_{n, 1}, \ldots, \sigma_{n, K}\right\} \leq \mathrm{CFL}
$$

holds for an appropriate number $\mathrm{CFL} \in(0,1)$. Here $\bar{\lambda}(U)$ denotes the maximal absolute value of all real eigenvalues of $D F(U), U \in \mathcal{U}_{1} \cup \mathcal{U}_{2}$. In the following calculations we used the CFL number 0.9.

3. Define for $i \in \mathbb{Z}$

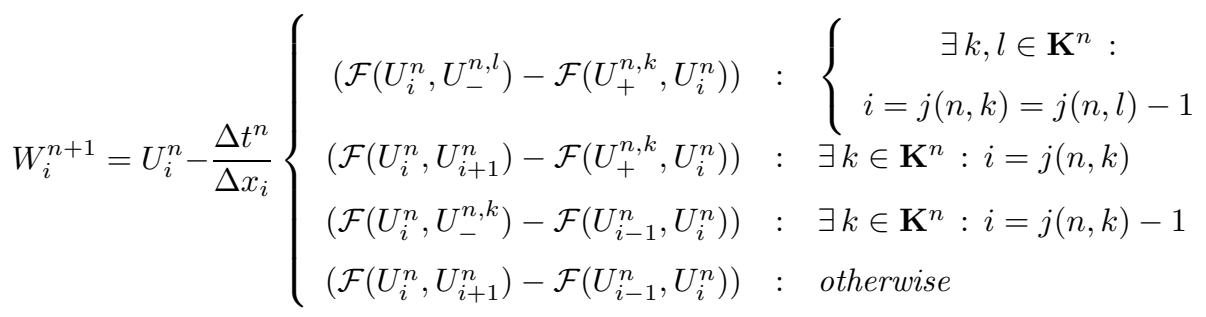

4. For $k \in \mathbf{K}^{n}$ define $\Gamma_{k}^{n+1}=\Gamma_{k}^{n}+\Delta t^{n} \sigma_{n, k}$ and for $i \in \mathbb{Z}$

$$
U_{i}^{n+1}=\left\{\begin{aligned}
\frac{1}{2}\left(U_{+}^{n, k}+U_{-}^{n, l}\right) & :\left\{\begin{array}{r}
\exists k, l \in \mathbf{K}^{n}: i=j(n, k)=j(n, l)-1 \\
\text { and } \Gamma_{k}^{n+1}>x_{i} \text { or } \Gamma_{l}^{n+1}<x_{i}
\end{array}\right. \\
U_{-}^{n, k} & : \exists k \in \mathbf{K}^{n}: i=j(n, k) \text { and } \Gamma_{k}^{n+1}>x_{i} \\
U_{+}^{n, k} & : \exists k \in \mathbf{K}^{n}: i=j(n, k)-1 \text { and } \Gamma_{k}^{n+1}<x_{i} \\
W_{i}^{n+1} & : \text { otherwise }
\end{aligned}\right.
$$

If the first case in (2.1) applies the corresponding indices in $\mathbf{K}^{n}$ are deleted, which finally defines $\mathbf{K}^{n+1}$.

In step 3 of Algorithm 2.1 the function $\mathcal{F}:\left(\mathcal{U}_{1} \cup \mathcal{U}_{2}\right)^{2} \rightarrow \mathbb{R}^{m}$ stands for an appropriate numerical flux for $F$ and $x_{i}$ in step 4 is the midpoint $\frac{1}{2}\left(x_{i-\frac{1}{2}}+x_{i+\frac{1}{2}}\right)$ of $T_{i}$.

We remark that the first case in steps $3 / 4$ corresponds to the case where a phase is extinguished (cf. in particular Section 3.4.3), the second and third case correspond to the advancement of the front by one cell, and finally the fourth case realizes the update in the pure bulk regions.

Remark 2.2 Note that Algorithm 2.1 is well-posed in the sense that the family $\left\{U_{i}^{n}\right\}_{i \in \mathbb{Z}}$ never takes values from $\mathcal{U}_{e}$.

Remark 2.3 Algorithm 2.1 is not conservative, cf. [23] for a discussion on the subject.

From Algorithm 2.1 we define the approximate solution

$$
U_{h}(x, t)=U_{i}^{n} \quad\left(x \in T_{i}, t \in\left[t^{n}, t^{n+1}\right) .\right.
$$

In the standard formulation of Algorithm 2.1 the exact solution of the Riemann problem is used. This requires a complex and time-consuming subroutine. In fact -depending on the number of phase boundaries and the mesh width- the Riemann solvers dominate the overall computing time by far. Therefore it is highly desirable to construct a cheap approximate solver. The construction of exact Riemann solutions and in particular its coding is quite complicated. Moreover, in many cases an exact Riemann solver is not available. This is our second motivation for the design of approximate solvers. 


\section{Exact and Approximate Riemann Solvers for Baro- tropic Materials}

In this section we introduce the new approximate Riemann solvers using the simple example of barotropic materials. In this case exact solvers for certain kinetic relations are known.

\subsection{The Mathematical Model and Exact Solvers}

We consider the system

$$
\begin{aligned}
& \partial_{t} u+\partial_{x} p(\tau)=0 \\
& \partial_{t} \tau-\partial_{x} u=0
\end{aligned}
$$

with pressure function $p(\tau)=-\Psi^{\prime}(\tau)$, where the smooth function $\Psi: \mathbb{R} \rightarrow \mathbb{R}$ is the free energy. The conserved variables $U=(u, \tau)^{T}$ are the particle velocity $u$ and the particle specific volume $\tau$. The eigenvalues $\lambda_{1}$ and $\lambda_{2}$ of system (3.1) are given by

$$
\lambda_{1}(U)=-\sqrt{-p^{\prime}(\tau)} \quad \text { and } \quad \lambda_{2}(U)=\sqrt{-p^{\prime}(\tau)} .
$$

Consequently system (3.1) is strictly hyperbolic, if and only if $p^{\prime}(\tau)<0$ for all $\tau \in \mathbb{R}$. The system is of hyperbolic-elliptic type, if we use a non-convex free energy function $\Psi$. Our standard example will be

$$
\Psi(\tau)=\frac{1}{4} \tau^{4}-\frac{1}{2} \tau^{2}
$$

For this example the system is strictly hyperbolic, if

$$
\tau \in \mathcal{Z}:=\mathcal{Z}_{1} \cup \mathcal{Z}_{2}:=\left(-\infty,-\sqrt{\frac{1}{3}}\right) \cup\left(\sqrt{\frac{1}{3}}, \infty\right) .
$$

So we have $\mathcal{U}_{1 / 2}=\mathbb{R} \times \mathcal{Z}_{1 / 2}$. The system (3.1) is equipped with the (mathematical) entropy $s(u, \tau)$ given by

$$
s(u, \tau)=\Psi(\tau)+\frac{1}{2} u^{2} .
$$

An entropy solution of (3.1) is a weak solution, which satisfies

$$
s(u, \tau)_{t}+(p(\tau) u)_{x} \leq 0
$$

in the sense of distributions. Assume $U_{0}: \mathbb{R} \rightarrow \mathcal{U}$ is given by

$$
U_{0}(x)= \begin{cases}U_{L}=\left(\tau_{L}, u_{L}\right)^{T} & \text { for } x<0 \\ U_{R}=\left(\tau_{R}, u_{R}\right)^{T} & \text { for } x>0 .\end{cases}
$$

If we consider a Riemann problem connecting states $U_{L}=\left(u_{L}, \tau_{L}\right)^{T}$ and $U_{R}=\left(u_{R}, \tau_{R}\right)^{T}$ in different hyperbolic domains, e.g.

$$
\tau_{L} \in \mathcal{Z}_{1} \quad \text { and } \quad \tau_{R} \in \mathcal{Z}_{2},
$$

then we assume, that solutions have the self-similar structure shown in Figure 1. The dashed lines symbolize the non-classical wave (phase boundary), the dotted lines stand for shock waves or rarefaction waves. $\sigma$ is the wave speed of the phase boundary. Without loss of generality we can assume $\sigma \geq 0$ in the following. This is due to the fact, that (3.1) is frame invariant.

As long as we are searching "only" for entropy solutions of (3.1), the problem is not wellposed for non-convex free energy functions. It is well known, that one has to supply an additional algebraic equation, which restricts the admissible non-classical waves. In the sequel we will call this equation kinetic relation following Abeyaratne\&Knowles [1]. We will write the kinetic relation in the form as in (1.5). 


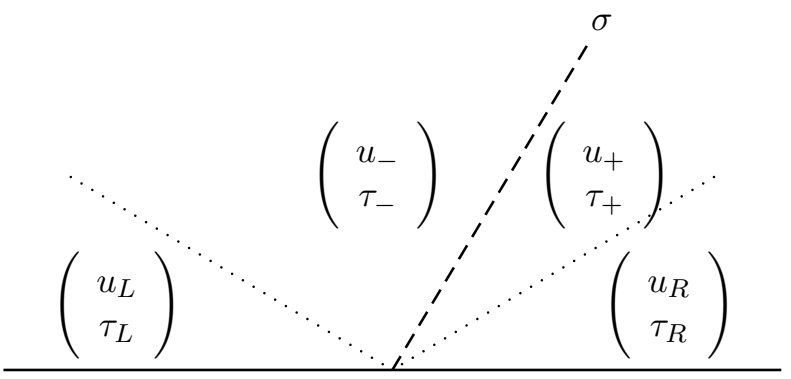

Figure 1: Typical wave structure of a non-classical Riemann solution of (3.1) with free energy (3.3).

Example 3.1 A simple but widely used kinetic relation associated to the energy (3.3) is the (piecewise) linear choice

$$
\mathcal{K}\left(U_{-}, U_{+}\right)=\kappa \tau_{-}-\operatorname{sign}\left(\tau_{-}\right) \frac{2 \kappa-1}{\sqrt{3}}+\tau_{+} \quad \text { with } \kappa \in\left[\frac{1}{2}, 1\right) .
$$

Then it is known, that the kinetic relation selects an unique entropy-consistent solution of the Riemann problem for end states in different phases. For details on an exact Riemann solver see [20, 21].

Example 3.2 We present an example from Abeyaratne\&KKnowles [3] of a non-linear kinetic relation. To define this kinetic relation we first introduce a so-called driving force $f$, which is given by

$$
f=f\left(\tau_{+}, \tau_{-}\right)=\Psi\left(\tau_{+}\right)-\Psi\left(\tau_{-}\right)+\frac{1}{2}\left(p\left(\tau_{+}\right)+p\left(\tau_{-}\right)\right)\left(\tau_{+}-\tau_{-}\right) .
$$

Using Rankine-Hugoniot conditions, the speed of the phase boundary $\sigma \in \mathbb{R}$, can be expressed in terms of $\tau_{+}$and $\tau_{-}$by

$$
\sigma=\sqrt{-\frac{p\left(\tau_{+}\right)-p\left(\tau_{-}\right)}{\tau_{+}-\tau_{-}}} .
$$

Then we can write the kinetic relation as

$$
\mathcal{K}\left(U_{-}, U_{+}\right)=V(f)-\sigma,
$$

where $V: \mathbb{R} \rightarrow \mathbb{R}$ is a constitutive function, which defines the wave speed in terms of the driving force. For the physical background see [3]. As an example for $V$ we will use in the numerical experiments

$$
V(f):=\operatorname{sign}(f) \sqrt{|f|}
$$

It can be shown, that any phase boundary, that satisfies (3.11) is an entropy solution in the sense of (3.6).

In this case it is known, that the kinetic relation does not select an unique solution for all Riemann problems. There are multiple solutions for Riemann problems of the form $U_{L}, U_{R} \in \mathcal{U}_{1}$ (or $U_{L}, U_{R} \in \mathcal{U}_{2}$ ). This can be resolved by the use of a nucleation criterion, see [20] and citations therein. In the setting $u_{L} \in \mathcal{Z}_{1}, u_{R} \in \mathcal{Z}_{2}$ (or vice versa) (3.11) selects a unique solution. 


\subsection{The Relaxation Riemann Solver}

We propose to approximate the solutions of the PDE model (3.1) with phase boundaries using a well-suited adaptation of a relaxation procedure introduced in the classical setting of the purely hyperbolic system, i.e. for $\Psi^{\prime \prime} \geq 0$ in (3.1).

Let us briefly recall, that within this simpler framework, the form of the relaxation model under consideration is

$$
\begin{aligned}
\partial_{t} \tau^{\lambda}-\partial_{x} u^{\lambda} & =0 \\
\partial_{t} u^{\lambda}+\partial_{x} \Pi^{\lambda} & =0 \\
\partial_{t} \Pi^{\lambda}+a^{2} \partial_{x} u^{\lambda} & =\lambda\left(p^{\lambda}-\Pi^{\lambda}\right)
\end{aligned}
$$

where $a>0$ is a given real number called the relaxation wave speed and $\lambda>0$ the relaxation parameter. Observe that in the regime of an infinite relaxation parameter $\lambda \rightarrow \infty$, we formally recover $\Pi=p(\tau)$, so that (3.13) boils down to the (purely hyperbolic) barotropic system. To prevent the relaxation procedure from instability, it is known, that the relaxation wave speed $a$ in (3.13) must satisfy the following Whitham-like condition

$$
a^{2}>\max _{\tau}\left(-p^{\prime}(\tau)\right),
$$

for all the $\tau$ under consideration. We refer the reader to $[5,9,8,17,26]$.

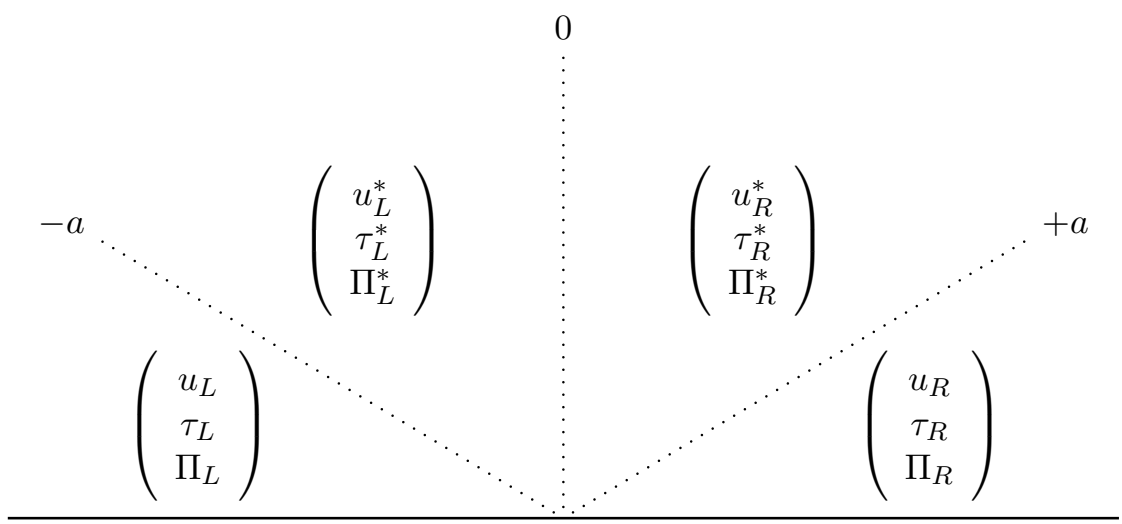

Figure 2: Typical wave structure of the Riemann solution for (3.15).

Let us also recall, that the solution of the proposed model relies on solving the Riemann problem for the homogeneous equations

$$
\begin{aligned}
& \partial_{t} \tau-\partial_{x} u=0, \\
& \partial_{t} u+\partial_{x} \Pi=0, \\
& \partial_{t} \Pi+a^{2} \partial_{x} u=0,
\end{aligned}
$$

with initial data at equilibrium:

$$
\tilde{U}_{0}(x)=\left(\tau_{0}, u_{0}, \Pi_{0}\right)(x)= \begin{cases}\tilde{U}_{L}=\left(\tau_{L}, u_{L}, \Pi_{L}=p\left(\tau_{L}\right)\right)^{T} & \text { for } \quad x<0 \\ \tilde{U}_{R}=\left(\tau_{R}, u_{R}, \Pi_{R}=p\left(\tau_{R}\right)\right)^{T} & \text { for } \quad x>0\end{cases}
$$


The classical Godunov method is then applied. A typical wave pattern of the Riemann solution is depicted in Figure 2 with intermediate states given by

$$
\begin{aligned}
u^{*}=u_{R}^{*}=u_{L}^{*} & =\frac{1}{2}\left(u_{R}+u_{L}\right)-\frac{1}{2 a}\left(\Pi_{R}-\Pi_{L}\right), \\
\Pi^{*}=\Pi_{R}^{*}=\Pi_{L}^{*} & =\frac{1}{2}\left(\Pi_{R}+\Pi_{L}\right)-\frac{a}{2}\left(u_{R}-u_{L}\right), \\
\tau_{L}^{*} & =\tau_{L}-\frac{1}{a}\left(u_{L}-u^{*}\right), \\
\tau_{R}^{*} & =\tau_{R}+\frac{1}{a}\left(u_{R}-u^{*}\right) .
\end{aligned}
$$

We refer for example to $[5,9]$ for the details.

The extension of the relaxation procedure to barotropic equations with phase boundaries is strongly motivated by the exact wave pattern depicted in Figure 1, namely with exactly one additional (transition) wave occurring in between the two extreme waves. To take into account this additional wave we propose to solve Riemann problems of the homogeneous equations (3.15), but with a singular perturbation:

$$
\begin{aligned}
\partial_{t} \tau & -\partial_{x} u=0 \\
\partial_{t} u+\partial_{x} \Pi & =0 \\
\partial_{t} \Pi+a^{2} \partial_{x} u & =\mathcal{M}\left(U_{L}, U_{R}\right) \delta_{x-\sigma\left(U_{L}, U_{R}\right) t}
\end{aligned}
$$

resulting from some Dirac-measure concentrated on the (approximate transition) wave propagating with speed $\sigma\left(U_{L}, U_{R}\right)$. In this paragraph we propose to predict $\sigma\left(U_{L}, U_{R}\right)$ according to

$$
\sigma\left(U_{L}, U_{R}\right)=-\frac{u_{R}-u_{L}}{\tau_{R}-\tau_{L}}
$$

and the corresponding mass $\mathcal{M}\left(U_{L}, U_{R}\right)$ according to the kinetic relation

$$
\mathcal{K}\left(U_{-}^{*}, U_{+}^{*}\right)=0,
$$

referring to the expected wave pattern with phase boundary depicted in Figure 3 (cf. Proposition 3.3 below).

Consider the special case of initial data (3.7) with

$$
\mathcal{K}\left(U_{L}, U_{R}\right)=0, \quad-s_{2}\left(u_{R}-u_{L}\right)+p_{R}-p_{L}=0 \quad \text { and } \quad s_{2}\left(\tau_{R}-\tau_{L}\right)+u_{R}-u_{L}=0 .
$$

Then the speed $\sigma\left(U_{L}, U_{R}\right)$ and the mass $\mathcal{M}\left(U_{L}, U_{R}\right)$ will be prescribed, such that any given isolated phase boundary transition in the exact Riemann problem for (3.1) with a data $U_{0}$ satisfying (3.7) and (3.20) is exactly preserved by the PDE model (3.18) with corresponding data given in $(3.16)$ built from $U_{0}$. Let us highlight, that the extended model (3.18) accounting for possible phase boundary transitions is solely addressed, when phase boundaries are likely to occur in the exact self similar solution, that is for data $U_{0}(x)$ involving two phases. We will more precisely focus on (3.8), the other cases being treated following similar steps. With such choice, we show

Proposition 3.3 Consider a kinetic relation $\mathcal{K}\left(U_{-}, U_{+}\right)=\tau_{+}-\varphi\left(\tau_{-}\right)$with strictly decreasing smooth function $\varphi: \mathcal{Z}_{1} \rightarrow \mathcal{Z}_{2}$. Let the initial datum $\tilde{U}_{0}$ be given as in (3.16) satisfying the phase boundary assumption (3.8) and $\sigma \neq 0$ with $\sigma$ defined in (3.19). Assume that the relaxation wave speed $a$ is chosen large enough.

Then there are states $U_{\#}^{*}, U_{-}^{*} \in \mathcal{U}_{1}$ and $U_{+}^{*} \in \mathcal{U}_{2}$, such that the self-similar function $\tilde{U}$ defined by the wave pattern in Figure 3 provides a distributional solution of (3.18) with $\sigma$ from (3.19) and

$$
\mathcal{M}\left(U_{L}, U_{R}\right)=-\sigma\left(a^{2}-\sigma^{2}\right)\left(\varphi\left(\tau_{-}^{*}\right)-\tau_{-}^{*}\right) .
$$

Furthermore $\tilde{U}$ is unique in the set of functions of this wave pattern. $\tilde{U}$ assumes the initial data from (3.16) in the weak sense. 


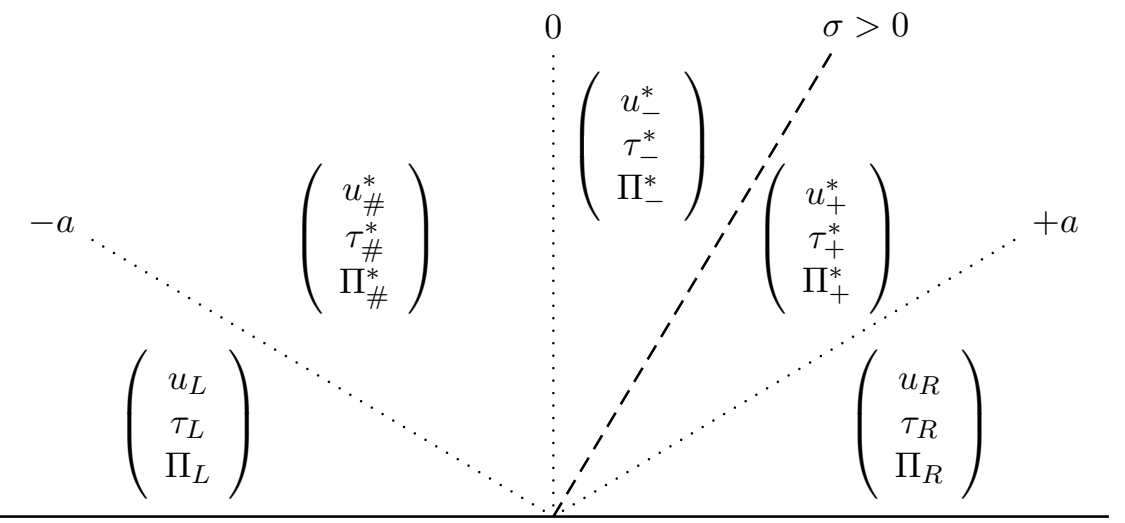

(a) $\sigma>0$

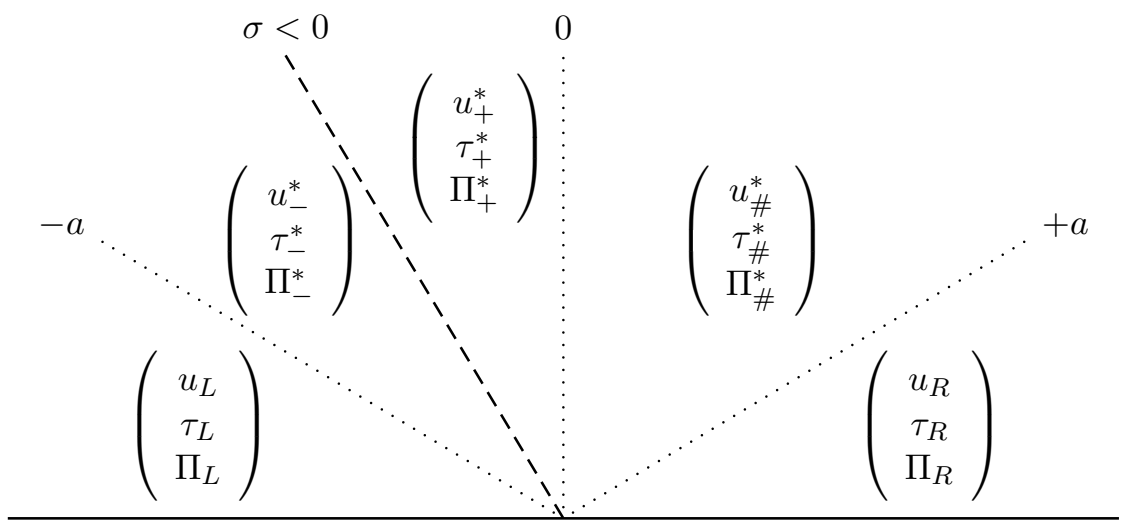

(b) $\sigma<0$

Figure 3: Typical wave pattern of Riemann solutions for (3.18).

Proof. Assume for the proof $\sigma>0$, the case $\sigma<0$ is done in the same way. The proof is done in two steps. First we follow the weak solution structure given in Figure 3 and set up a system of 8 linear and one non-linear equations for the unknown states displayed in Figure 3. Then we show, that the system we found is uniquely solvable.

By construction (3.18) has only solutions of the form given in Figure 3. Considering the Rankine-Hugoniot conditions for the different waves we find for the waves travelling with speed $-a$ and $+a$

$$
\begin{aligned}
& \Pi_{L}+a u_{L}=\Pi_{\#}^{*}+a u_{\#}^{*}, \\
& \Pi_{L}+a^{2} \tau_{L}=\Pi_{\#}^{*}+a^{2} \tau_{\#}^{*}, \\
& \Pi_{R}-a u_{R}=\Pi_{+}^{*}-a u_{+}^{*}, \\
& \Pi_{R}+a^{2} \tau_{R}=\Pi_{+}^{*}+a^{2} \tau_{+}^{*} .
\end{aligned}
$$

The conditions for the stationary wave are

$$
u_{\#}^{*}=u_{-}^{*} \quad \text { and } \quad \Pi_{\#}^{*}=\Pi_{-}^{*} .
$$

From the wave given by the Dirac measure we get the restrictions

$$
\begin{aligned}
& \sigma\left(\tau_{+}^{*}-\tau_{-}^{*}\right)=-\left(u_{+}^{*}-u_{-}^{*}\right), \\
& \sigma\left(u_{+}^{*}-u_{-}^{*}\right)=\Pi_{+}^{*}-\Pi_{-}^{*}, \\
& \sigma\left(\Pi_{+}^{*}-\Pi_{-}^{*}\right)=a^{2}\left(u_{+}^{*}-u_{-}^{*}\right)-\mathcal{M}\left(U_{L}, U_{R}\right) .
\end{aligned}
$$


The last equation of $(3.23)$ is equivalent to

$$
\tau_{+}^{*}=\varphi\left(\tau_{-}^{*}\right)
$$

by using the definition of $\mathcal{M}\left(U_{L}, U_{R}\right)$ and the first two equations in (3.23). If $\varphi$ is linear for instance take (3.9), then the whole set of equations becomes linear with determinant given by

$$
a^{3}\left(2 \kappa a^{2}-(1+\kappa)\left(a \sigma+\sigma^{2}\right)\right)>0 \quad\left(\frac{1}{2} \leq \kappa \leq 1\right),
$$

provided $a$ is sufficient large. We thus have existence and uniqueness in the class of functions with wave pattern displayed in Figure 3.

In the non-linear case we can solve the linear part and reduce the problem to a scalar equation for the remaining unknown $\tau_{-}^{*}$ in the form

$$
\varphi\left(\tau_{-}^{*}\right)=C(a) \tau_{-}^{*}+D(a)
$$

Here $C(a)$ and $D(a)$ are given by

$$
C(a)=-\frac{\sigma^{2}+\sigma a}{2 a^{2}-\sigma a-\sigma^{2}} \quad \text { and } \quad D(a)=\frac{p\left(\tau_{R}\right)-p\left(\tau_{L}\right)+a\left(u_{R}-u_{L}\right)+2 a^{2} \tau_{R}}{2 a^{2}-\sigma a-\sigma^{2}} .
$$

For $a \rightarrow \infty$ we get

$$
C(a)<0, \quad C(a) \rightarrow 0 \quad \text { and } \quad D(a) \rightarrow \tau_{R}
$$

With these properties and $\varphi^{\prime}<0$ we conclude, that the function given by

$$
\tau \mapsto \varphi^{-1}(C(a) \tau+D(a))
$$

is a contraction for $a$ large enough and thus has a unique fixed point. In other words we get a unique solution of the equations (3.21), (3.22) and (3.23).

The following consistency result for isolated phase boundaries is a direct consequence of Proposition 3.3.

Corollary 3.4 Let $\tilde{U}_{0}$ be given as described in (3.16) satisfying (3.20), so that the exact Riemann solution for (3.1) is made of a single phase boundary travelling with speed $\sigma\left(U_{L}, U_{R}\right)$. Assume further, that the chosen kinetic relation selects a unique solution. Then the Relaxation Riemann (RR) solution of (3.18) given in Proposition 3.3 reads

$$
\tilde{U}\left(\frac{x}{t}\right)=\left\{\begin{array}{cl}
\tilde{U}_{L} & : \quad x<\sigma\left(U_{L}, U_{R}\right) t \\
\tilde{U}_{R} & : \quad x>\sigma\left(U_{L}, U_{R}\right) t
\end{array}\right.
$$

Therefore, the first two components of $\tilde{U}$ coincide with the exact Riemann solution of (3.1).

It remains to study the behavior of the Relaxation Riemann (RR) solver at $\sigma=0$. Let us understand the RR solver from Proposition 3.3 as a mapping

$$
P^{\prime}:\left(u_{L}, \tau_{L}, u_{R}, \tau_{R}\right) \mapsto\left(u_{-}^{*}, \tau_{-}^{*}, u_{+}^{*}, \tau_{+}^{*}\right) .
$$

This definition of the RR solver is not continuous at $\sigma=0$, since we have in the limit $\varphi\left(\tau_{-}^{*}\right)=\tau_{+}^{*}$ for a sequence of initial data satisfying $\sigma>0$, but $\varphi\left(\tau_{+}^{*}\right)=\tau_{-}^{*}$ for $\sigma<0$.

The reason for the discontinuity is the wave pattern. We assumed for $\sigma>0$ in total 4 waves, but at $\sigma=0$ they degenerate to 3 waves. A solver of the form (for $\sigma>0$ )

$$
P^{\prime \prime}:\left(u_{L}, \tau_{L}, u_{R}, \tau_{R}\right) \mapsto \begin{cases}\left(u_{\#}^{*}, \tau_{\#}^{*}, u_{+}^{*}, \tau_{+}^{*}\right) & \text { for } \sigma>0, \\ \left(u_{-}^{*}, \tau_{-}^{*}, u_{\#}^{*}, \tau_{\#}^{*}\right) & \text { for } \sigma<0\end{cases}
$$


would be much more accurate for $\sigma \rightarrow 0$ and continuous at $\sigma=0$.

The numerical experiments in the rest of the paper rely on the following defintion of a relaxation Riemann solver:

Definition 3.5 Let $\delta: \mathbb{R} \rightarrow[0,1]$ be a continuous function with $\lim _{\sigma \rightarrow \pm \infty} \delta(\sigma)=0$ and $\delta(0)=1$. The mapping

$$
P:\left(u_{L}, \tau_{L}, u_{R}, \tau_{R}\right) \mapsto(1-\delta(\sigma)) P^{\prime}\left(u_{L}, \tau_{L}, u_{R}, \tau_{R}\right)+\delta(\sigma) P^{\prime \prime}\left(u_{L}, \tau_{L}, u_{R}, \tau_{R}\right),
$$

is called $\mathbf{R}$ elaxation $\mathbf{R}$ iemann $(R R)$ solver. In the following numerical experiments we will use

$$
\delta(\sigma)=\frac{1}{e-1}\left(\exp \left(\frac{1}{1+100 \sigma^{2}}\right)-1\right) .
$$

\subsubsection{A Numerical Experiment: Basic Performance}

We test the RR solver plugged into Algorithm 2.1 from Section 2. Instead of the trace values of the exact Riemann solver, namely $U_{-}$and $U_{+}$, we now use the values $U_{-}^{*}$ and $U_{+}^{*}$, which are calculated by the relaxation Riemann solver from Definition (3.5). In Figure 4 we plotted the $L^{1}\left((0,0.1) ; L^{1}(-1,1)\right)$ and $L^{1}\left((0,0.1) ; L^{2}(-1,1)\right)$-errors for four Riemann problems (named RP1, RP2, RP3 and RP4). The four Riemann problems are

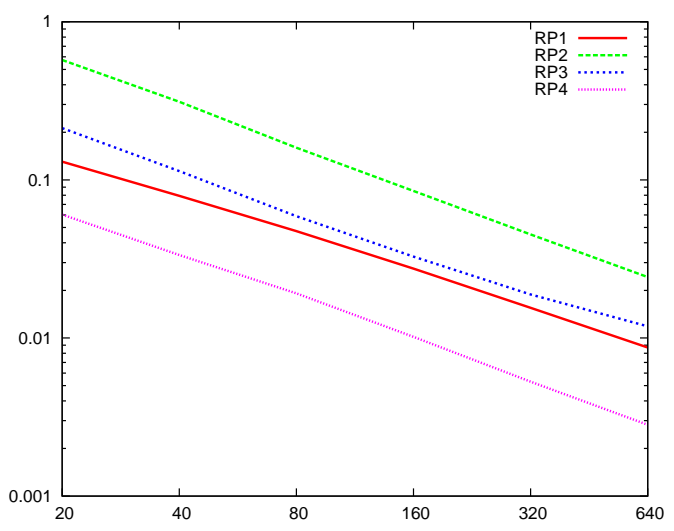

(a) $L^{1}\left(L^{1}\right)$-Error.

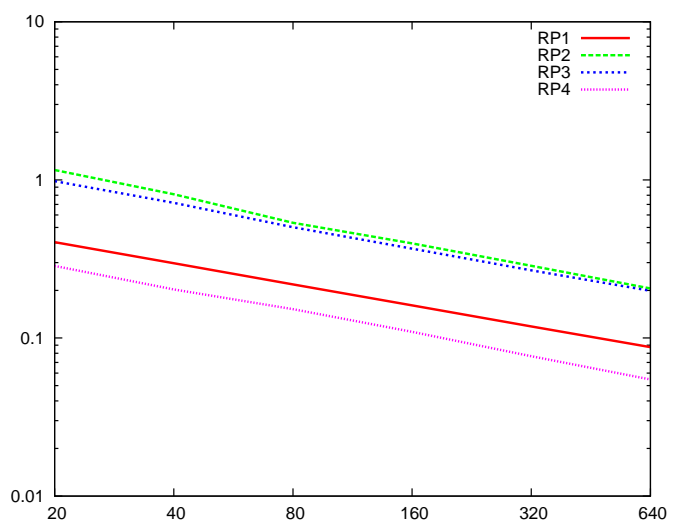

(b) $L^{1}\left(L^{2}\right)$-Error.

Figure 4: Error for different Riemann problems solved with the RR solver on uniform meshes with 20 to 640 cells.

$\mathrm{RP} 1: \quad U_{L}=\left(\begin{array}{c}0 \\ -1\end{array}\right), U_{R}=\left(\begin{array}{l}0 \\ 2\end{array}\right)$

RP3: $U_{L}=\left(\begin{array}{c}0 \\ -4\end{array}\right), U_{R}=\left(\begin{array}{c}-7 \sqrt{12} \\ 3\end{array}\right)$
$\mathrm{RP2}: \quad U_{L}=\left(\begin{array}{c}0 \\ -3\end{array}\right), U_{R}=\left(\begin{array}{c}-7 \sqrt{12} \\ 3\end{array}\right)$ RP4: $\quad U_{L}=\left(\begin{array}{c}0 \\ -1.2\end{array}\right), U_{R}=\left(\begin{array}{c}0.5 \\ 1.2\end{array}\right)$.

We use the linear kinetic relation from Example 3.1 with $\kappa=\frac{3}{4}$. All different wave types appear in the set of Riemann problems: RP3 is an isolated phase boundary, RP1 contains a rarefaction wave, a phase boundary and a shock wave. One can clearly see, that the tracking-type algorithm converges. The experimental order of accuracy is approximately 0.8 for the $L^{1}\left(L^{1}\right)$-norm and 0.45 for the $L^{1}\left(L^{2}\right)$-norm.

The RR solver based scheme seems to converge. But in terms of efficiency we will see in Section 3.4.2, that the RR solver is not preferable in contrast to an exact Riemann solver known for this simple model. By efficiency, we again mean the run-time required to stay 
below a prescribed error threshold. In the following subsections we suggest therefore two iterative procedures, which use the RR solution given by (3.21), (3.22) and (3.23) and (3.19) and produce a more accurate solution. We will see in Section 3.4.2, that from an efficiency point of view, these approaches should be favored.

\subsection{Enhancing the Relaxation Riemann Solver by Sub-Iteration}

Algorithm 2.1 requires values for traces at phase boundaries as they appear in solutions of Riemann problems. In the previous Section 3.2 we introduced the approximative RR solver and used traces from the RR solution at the phase boundary in Algorithm 2.1. In this section we will propose a sub-iteration technique to improve the quality of the RR approximation. It turns out that these enhanced approximations finally lead to the most efficient variants of Algorithm 2.1.

The sub-iteration follows the subsequent general concept. Consider the Riemann problem (3.1), (3.7) and the corresponding RR solution with trace values $U_{ \pm}^{*}$ depending on $U_{L}, U_{R}$. For the sub-iteration procedure we will introduce mappings $Q: \mathcal{U}_{1} \times \mathcal{U}_{2} \rightarrow \mathcal{U}_{1} \times \mathcal{U}_{2}$. A sequence of approximate trace values $\left\{\left(U_{-}^{*, l}, U_{+}^{*, l}\right)\right\}_{l \in \mathbb{N}_{0}}$ is then obtained by the formula

$$
\left(\begin{array}{c}
U_{-}^{*, l+1} \\
U_{+}^{*, l+1}
\end{array}\right)=Q\left(\begin{array}{c}
U_{-}^{*, l} \\
U_{+}^{*, l}
\end{array}\right), \quad\left(\begin{array}{c}
U_{-}^{*, 0} \\
U_{+}^{*, 0}
\end{array}\right)=\left(\begin{array}{c}
U_{L} \\
U_{R}
\end{array}\right) .
$$

In the sequel we suggest two choices for mappings $Q$ which lead to two different iterative procedures. It is interesting to note that one of the constructed sequences $\left\{\left(U_{-}^{*, l}, U_{+}^{*, l}\right)\right\}_{l \in \mathbb{N}_{0}}$ seems to converge even towards the exact traces $\left(U_{-}, U_{+}\right)$as the number of sub-iterations increases (cf. Example 3.9 in Section 3.3.2).

In what follows it is convenient to use the functional notations

$$
U_{ \pm}^{*}=P_{ \pm}\left(U_{L}, U_{R}\right)
$$

for the approximate phase boundary traces computed by the RR solver $P$ from (3.27) together with and as before

$$
\sigma=\sigma\left(U_{L}, U_{R}\right)=-\frac{u_{R}-u_{L}}{\tau_{R}-\tau_{L}}
$$

For all numerical experiments using (3.28) the iteration is stopped if either the difference from last iteration is smaller than some tolerance $\varepsilon>0$ or the iteration count is less than a given integer $N$.

\subsubsection{Sub-Iteration with Numerical Fluxes}

Let us denote the flux in $(3.1)$ by $F(U)=(p(\tau),-u)^{T}$. For some $r \in \mathbb{R}$ let $\mathcal{F}_{r}:\left(\mathcal{U}_{1} \times\right.$ $\left.\mathcal{U}_{1}\right) \cup\left(\mathcal{U}_{2} \times \mathcal{U}_{2}\right) \rightarrow \mathbb{R}^{m}$ be a numerical flux function consistent with the shifted flux $F_{r}(U):=F(U)-r U$. In the present work, a Lax-Friedrichs type of numerical flux function is chosen :

$$
\mathcal{F}_{r}(V, W)=\frac{1}{2}\left(\mathcal{F}_{r}(V)+F_{r}(W)\right)+\frac{1}{2 \gamma}(V-W), \quad V, W \in\left(\mathcal{U}_{1} \times \mathcal{U}_{1}\right) \cup\left(\mathcal{U}_{2} \times \mathcal{U}_{2}\right) .
$$

Here we used $\gamma=\frac{1}{2}\left(\max \left\{\lambda_{2}(V), \lambda_{2}(W)\right\}+|r|\right)^{-1}$. Now, for given number $\alpha>0$, iterates are computed by applying (3.28) with the mapping

$$
Q\left(\begin{array}{c}
U_{1} \\
U_{2}
\end{array}\right)=\left(\begin{array}{c}
U_{1}+\alpha\left(\mathcal{F}_{\sigma}\left(U_{1}, U_{1}\right)-\mathcal{F}_{\sigma}\left(U_{1}, P_{-}\left(U_{1}, U_{2}\right)\right)\right. \\
U_{2}+\alpha\left(\mathcal{F}_{\sigma}\left(P_{+}\left(U_{1}, U_{2}\right), U_{2}\right)-\mathcal{F}_{\sigma}\left(U_{2}, U_{2}\right)\right)
\end{array}\right),
$$


with $\sigma$ given in (3.29). If (3.20) holds, the pair $\left(U_{L}, U_{R}\right)$ is a fixed point for (3.28), (3.30). The mapping $Q$ mimics a finite-volume scheme on a "two-cell micro-scale mesh". The free constant $\alpha>0$ is used to trigger a stable sequence similar to stabilizing an explicit finite-volume scheme by Courant-Friedrichs-Levy conditions.

In the following we will call this approach Relaxation Riemann solver with sub-iterations using a Finite Volume approach, short RR-sub-FV.

Remark 3.6 The RR-sub-FV solver does not use particular properties of the barotropic system (3.1). Therefore it can be applied in connection with an appropriate approximate Riemann solver to initial value problems for arbitrary systems (1.1). We refer to Section 4 for an application in the framework of non-barotropic materials.

\subsubsection{Sub-Iteration in State Space}

For this approach we rely on wave curves emerging from the states $U_{L}, U_{R}$ in the twodimensional state space $\mathcal{U}$. It uses some structural properties of the barotropic system (3.1) which we review in short. It is well-known [18], that the forward 1-rarefaction curve starting in $U_{L} \in \mathcal{U}_{1}$ can be parameterized using the specific volume $\tau$ for $\tau \geq \tau_{L}$. It is expressed by

$$
u^{\prime}(\tau)=-\sqrt{-p^{\prime}(\tau)} \text { and } u\left(\tau_{L}\right)=u_{L} .
$$

The forward Rankine-Hugoniot locus for 1-shock waves satisfies

$$
u(\tau)=u_{L}+\frac{1}{s\left(\tau_{L}, \tau\right)}\left(p(\tau)-p\left(\tau_{L}\right)\right), \quad s\left(\tau_{L}, \tau\right)=-\sqrt{-\frac{p(\tau)-p\left(\tau_{L}\right)}{\tau-\tau_{L}}}, \quad \tau \leq \tau_{L}
$$

The description of the forward 1-waves -regardless whether rarefaction or shock wave but including entropy admissibility- can then be rewritten in the compact form using generalized eigenvectors:

$$
U_{1}^{\prime}(\tau)=g_{1}\left(\tau_{L}, \tau\right):=\left(\begin{array}{c}
\frac{1}{2}\left(\frac{p^{\prime}(\tau)}{s_{1}\left(\tau_{L}, \tau\right)}-s_{1}\left(\tau_{L}, \tau\right)\right) \\
1
\end{array}\right), \quad U\left(\tau_{L}\right)=U_{L}
$$

Here $s_{1}$ is given by

$$
s_{1}=s_{1}\left(\tau_{L}, \tau\right)= \begin{cases}-\sqrt{-p^{\prime}(\tau)} & \text { for } \tau \geq \tau_{L}, \\ -\sqrt{-\frac{p(\tau)-p\left(\tau_{L}\right)}{\tau-\tau_{L}}} & \text { for } \tau<\tau_{L} .\end{cases}
$$

In case of backward 2-curves emanating from $U_{R}$ a similar compact form for the parameterization can be obtained:

$$
U_{2}^{\prime}(\tau)=g_{2}\left(\tau, \tau_{R}\right)=\left(\begin{array}{c}
\frac{1}{2}\left(\frac{p^{\prime}(\tau)}{s_{2}\left(\tau, \tau_{R}\right)}-s_{2}\left(\tau, \tau_{R}\right)\right) \\
1
\end{array}\right), \quad U\left(\tau_{R}\right)=U_{R}
$$

with

$$
s_{2}=s_{2}\left(\tau, \tau_{R}\right)= \begin{cases}\sqrt{-p^{\prime}(\tau)} & \text { for } \tau \geq \tau_{R} \\ \sqrt{-\frac{p\left(\tau_{R}\right)-p(\tau)}{\tau_{R}-\tau}} & \text { for } \tau<\tau_{R}\end{cases}
$$

To introduce the sub-iteration let us now consider for $\xi \geq 0$ the ordinary initial value problem

$$
\left(\begin{array}{c}
U_{1}^{\prime}(\xi) \\
U_{2}^{\prime}(\xi)
\end{array}\right)=\left(\begin{array}{c}
\left(\tau_{-}^{*}(\xi)-\tau_{1}(\xi)\right) g_{1}\left(\tau_{L}, \tau_{1}(\xi)\right) \\
\left(\tau_{+}^{*}(\xi)-\tau_{2}(\xi)\right) g_{2}\left(\tau_{2}(\xi), \tau_{R}\right)
\end{array}\right), \quad\left(\begin{array}{c}
U_{1}(0) \\
U_{2}(0)
\end{array}\right)=\left(\begin{array}{c}
U_{L} \\
U_{R}
\end{array}\right)
$$


with unknowns $U_{1}(\xi)=\left(u_{1}(\xi), \tau_{1}(\xi)\right)^{T}$ and $U_{2}(\xi)=\left(u_{2}(\xi), \tau_{2}(\xi)\right)^{T}$. Note that $\tau_{-}^{*}(\xi)$ and $\tau_{+}^{*}(\xi)$ are the $\tau$-components of $P_{ \pm}\left(U_{1}(\xi), U_{2}(\xi)\right)$ given by the RR solver.

Using Corollary 3.4 it is obvious that any pair of states $\left(U_{-}, U_{+}\right)$that satisfies the kinetic relation (1.5) is a rest point of (3.33). Since both $g_{1}$ and $g_{2}$ never vanish the set of rest points is given exactly by the set of states that solve (1.5). It is hard to see that the flow of (3.33) is (globally) attractive but at least we can present a consistency result.

Proposition 3.7 For $\left(U_{L}, U_{R}\right) \in \mathcal{U}_{1} \times \mathcal{U}_{2}$ assume that (3.1), (3.7) has a unique solution with phase boundary traces $U_{-}$and $U_{+}$that satisfy a kinetic relation (1.5)(see Figure 1 ). Furthermore let there be a global solution $\left(U_{1}, U_{2}\right):[0, \infty) \rightarrow \mathcal{U}_{1} \times \mathcal{U}_{2}$ of (3.33) and a pair $\left(W_{-}, W_{+}\right) \in \mathcal{U}_{1} \times \mathcal{U}_{2}$ such that

$$
\left(\begin{array}{l}
W_{-} \\
W_{+}
\end{array}\right)=\lim _{\xi \rightarrow \infty}\left(\begin{array}{c}
U_{1}(\xi) \\
U_{2}(\xi)
\end{array}\right)
$$

holds.

Then we have $W_{ \pm}=U_{ \pm}$.

Proof. By construction the state $U_{L}$ is connected by a forward 1-wave to $U_{1}(\xi)$ for all $\xi>0$, thus by (3.34) also to $W_{-}$. In the same way a backward 2-wave connects $U_{R}$ with $W_{+}$. The proof is now a direct consequence of Corollary 3.4.

Finally we can define iteration mappings $Q$ as needed in (3.28) by discretizing the initial value problem (3.33). In the numerical experiments a fourth order Runge-Kutta method is used. In the following the step size is denoted by $\Delta \xi$. We will call the method Relaxation Riemann solver with sub-iterations using Generalized Eigenvectors, or for short RR-subGE.

Remark 3.8 Instead of generalized eigenvectors we can also use eigenvectors in (3.33). In this case one cannot expect to converge to the correct solution as analyzed above in Proposition 3.7. However embedding this approach into Algorithm 2.1 leads to good results, see numerical experiments in Section 3.4.1. This method is called Relaxation Riemann solver with sub-iterations using Eigenvectors, or for short RR-sub-E.

Table 1: Mean and maximum errors comparing the RR-sub-GE approach to trace values of the exact Riemann solution for different parameter sets.

\begin{tabular}{cccccccc}
$\Delta \xi$ & $\varepsilon$ & $N$ & av. $N$ & $\operatorname{mean} u$ & $\max u$ & $\operatorname{mean} \tau$ & $\max \tau$ \\
\hline 0.4 & $10^{-2}$ & 25 & 11.8 & 0.175147 & 3.300120 & 0.021796 & 0.201660 \\
0.2 & $10^{-3}$ & 50 & 36.9 & 0.015614 & 0.301569 & 0.007902 & 0.078560 \\
0.1 & $10^{-4}$ & 200 & 117. & 0.005125 & 0.021660 & 0.005244 & 0.057971 \\
\hline
\end{tabular}

Example 3.9 In Table 1 we study the convergence properties of the RR-sub-GE solver what concerns the trace values at the phase boundaries. The dependence on the iteration parameters $\Delta \xi, \varepsilon$ and $N$ is illustrated.

We use the linear kinetic relation (3.9) with $\kappa=\frac{3}{4}$. Without loss of generality we choose $u_{L}=0$. The other variables are chosen from $u_{R} \in[-1,1], \tau_{L} \in[-2,-0.7]$ and $\tau_{R} \in[0.7,2]$ in an equidistant way to get $J:=1331$ different Riemann problems. Due to the fact, that $\tau_{L}$ and $\tau_{R}$ lie in different phases, we always deal with Riemann solutions with phase boundary. Finally we calculate the mean and the maximum error over this set, which are defined by

$$
\max \tau:=\max _{1 \leq j \leq J}\left|\tau_{-(j)}^{*}-\tau_{-(j)}\right|+\left|\tau_{+(j)}^{*}-\tau_{+(j)}\right|
$$


and

$$
\text { mean } \tau:=\frac{1}{J} \sum_{1 \leq j \leq J}\left|\tau_{-(j)}^{*}-\tau_{-(j)}\right|+\left|\tau_{+(j)}^{*}-\tau_{+(j)}\right| .
$$

The index $j \in J$ parameterizes the different examples. Analogous definitions are used for max $u$ and mean $u$. The column "av. N" gives the average number of iterations needed to satisfy one of the stopping criteria.

This numerical result and Proposition 3.7 are strong arguments, that the RR-sub-GE solver leads to convergence to the trace values of the exact Riemann solution!

\subsection{Numerical Experiments}

In this section we test the approximate Riemann solvers RR, RR-sub-FV, RR-sub-GE and RR-sub-E with respect to accuracy and computational efficiency when used in Algorithm (2.1) to solve arbitrary initial value problems. The first experiment is an exception as it is devoted to the comparison of the approximation quality for phase boundary trace values.

\subsubsection{Approximate Riemann Solvers and Traces at the Phase Bound- ary}

Table 2: Mean and maximum errors for different parameter sets.

(a) In this experiment we used $\Delta \xi=0.2, \varepsilon=10^{-3}$ and $N=50$.

\begin{tabular}{lcccc} 
& $\operatorname{mean} u$ & $\max u$ & $\operatorname{mean} \tau$ & $\max \tau$ \\
\hline RR & 1.482390 & 7.224300 & 1.809490 & 10.000000 \\
RR-sub-FV & 1.125550 & 9.652720 & 0.297517 & 1.257820 \\
RR-sub-GE & 0.015614 & 0.301569 & 0.007902 & 0.078560 \\
RR-sub-E & 0.015718 & 0.301569 & 0.007614 & 0.078560 \\
\hline
\end{tabular}

(b) In this experiment we used $\Delta \xi=0.1, \varepsilon=10^{-3}$ and $N=200$.

\begin{tabular}{lcccc} 
& $\operatorname{mean} u$ & $\max u$ & $\operatorname{mean} \tau$ & $\max \tau$ \\
\hline RR & 1.482390 & 7.224300 & 1.809490 & 10.000000 \\
RR-sub-FV & 1.082170 & 9.651910 & 0.298076 & 1.258090 \\
RR-sub-GE & 0.005125 & 0.021660 & 0.005244 & 0.057971 \\
RR-sub-E & 0.005296 & 0.021660 & 0.004954 & 0.057971 \\
\hline
\end{tabular}

We compare the trace values at phase boundaries given by the different approximate Riemann solvers proposed in the last sections. To this end we build the exact Riemann solution along with the self-similar functions coming out of the proposed approximate Riemann solvers and then compare point-wisely the resulting traces. The experimental set-up and notations are identical to the ones in Example 3.9.

Note that what is expected is an improvement of the accuracy when using the iterative procedure, but not at all a convergence of the approximate trace values to the traces of the phase boundary of the exact Riemann problem solution for all approaches.

From Table 2 (and Example 3.9) it seems to be clear, that we should prefer to use the RR-sub-GE method in Algorithm 2.1. But in the following we will see, that RR-sub-FV approach can be applied in more general settings and gives indeed comparable results. We conclude with a minor comment on the results displayed in Table 2: If the 1-wave and the 2-wave are rarefaction waves, the methods RR-sub-GE and RR-sub-E coincide. In Table 2(a) the maximal error of the two methods (both using the same fourth order Runge-Kutta method) matches perfectly. Probably the Riemann data corresponding to 
the computations with the maximal error leads to a solution containing two rarefaction waves.

\subsubsection{Tracking Algorithm 2.1 with Approximate Riemann Solvers for Two-Phase Initial Datum}

In this section we want to test Algorithm 2.1 together with the approximate Riemann solvers, we have constructed. As a test problem we consider again a Riemann problem on the computational domain $(-1,1)$ with

$$
U_{L}=\left(\begin{array}{r}
0.0 \\
-1.2
\end{array}\right) \quad \text { and } \quad U_{R}=\left(\begin{array}{r}
-0.5 \\
1.0
\end{array}\right)
$$

and a linear kinetic relation (3.9) with $\kappa=\frac{3}{4}$.

In Figure 5 one can clearly see the differences between the RR solver and the enhanced methods. The RR solver produces a "non-monotonic" solution, but all other methods do not have over- or under-shoots at the 1- and 2-waves. Between the enhanced methods there is no eye-catching difference.

Energy/Entropy Stability: For the same experiment we also measured the time evolution of the total energy/entropy in the system, which is given by

$$
E_{h}(t)=\int_{-1}^{1} s\left(u_{h}(x, t), \tau_{h}(x, t)\right) \mathrm{d} x+p\left(\tau_{h}(1, t)\right) u_{h}(1, t)-p\left(\tau_{h}(-1, t)\right) u_{h}(-1, t)
$$

with $U_{h}=\left(u_{h}, \tau_{h}\right)^{T}$ defined as in (2.2). Figure 6 shows the result - a monotonic decaying function in time.

Two additional points deserve attention. For the RR solver initially there is a short time span, which the algorithm needs to get close to the correct solution. After that time span the error lines for all the proposed methods stay parallel. They arise at times when the algorithm pushes the phase boundary one cell further.

Run-Time Comparison: In Figure 7 we compare the run-time of the overall method for the different Riemann solvers. The relaxation Riemann solver (RR) is only for coarse grids a good choice. For fine grids all other Riemann solvers are clearly superior. The enhanced approximate Riemann solvers produce approximately the same errors, but differ in runtime. This difference gets smaller with finer grids, since the work needed for the finite-volume scheme in the bulk phases increases faster, than work on the Riemann problem. The RR-sub-FV solver seems to be the fastest one.

\subsubsection{Tracking Algorithm 2.1 with Approximate Riemann Solvers for Phase-Distincting Initial Datum}

Let us interpret (3.1) as a model for an elastic bar, i.e. $\tau$ stands for strain and $-p$ for the stress function. Although Algorithm 2.1 is not capable of phase nucleation, phase distinction is possible. In our experiment with the RR solver we assume an elastic bar to be fixed at the top and a weight to be is installed at the bottom. In Figure 8 we can see the length of the elastic bar evolving in time. The colors indicate the different phases. We compare different linear kinetic relations and different weights. We use linear kinetic relations of the form $\mathcal{K}\left(U_{L}, U_{R}\right)=\kappa \tau_{L}+\tau_{R}$ or $\kappa \in\left\{\frac{1}{2}, \frac{9}{10}\right\}$. Initially $u=0$ and $\tau \in\{-1,1\}$ with in total 4 phase boundaries. At the upper boundary we use the conditions $u=0$ and $\tau^{\prime}=0$. At the lower boundary we use $u^{\prime}=0$ and $p(\tau)=p_{0}$, where $p_{0}=0.32$ for the low weight and $p_{0}=0.5$ for the heavy weight.

The experiment shows that phase distinction can be simulated by our approach. It depends sensitively on the choice of kinetic relation. 

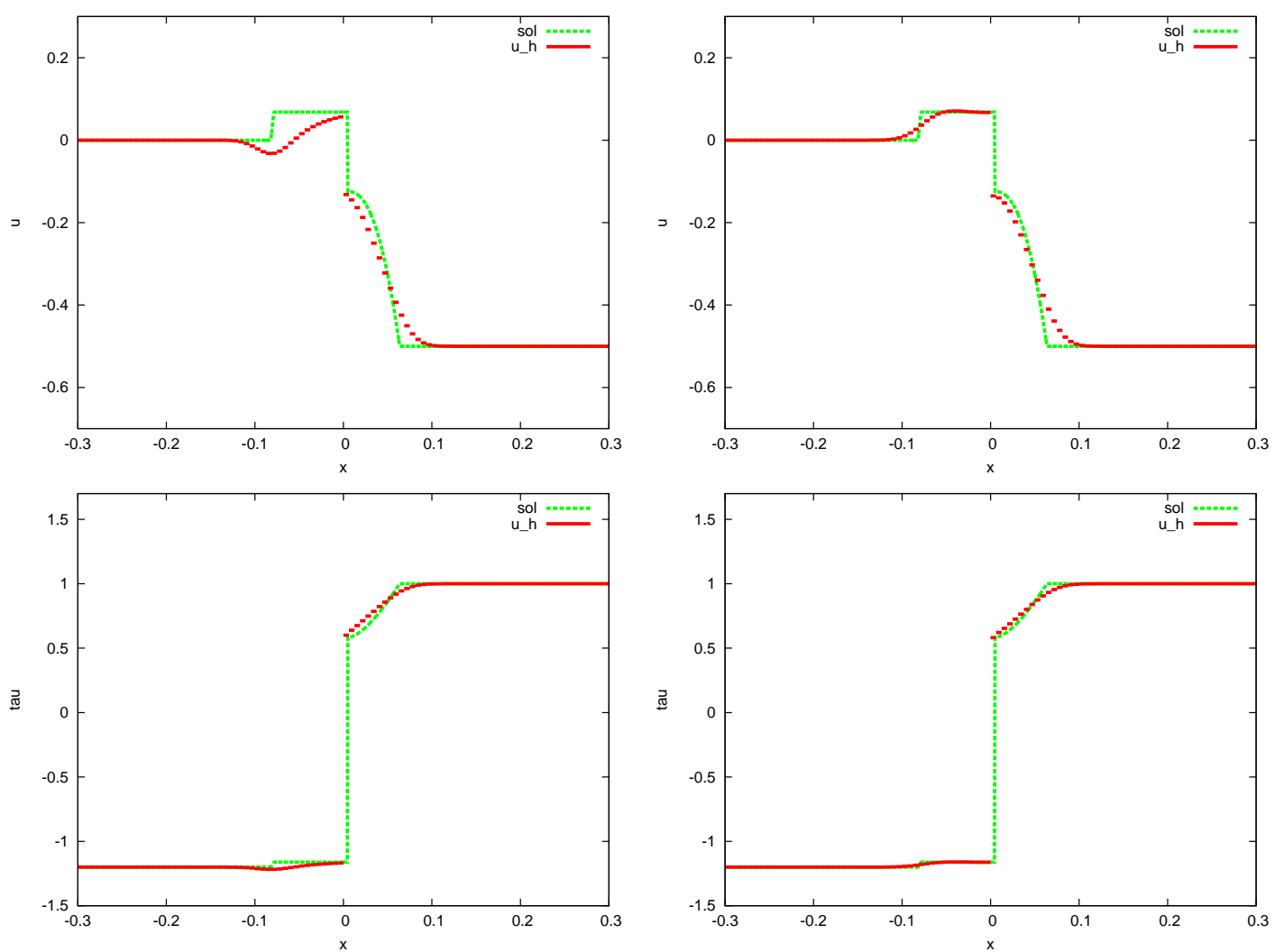

(a) $\mathrm{RR}$

(b) RR-sub-FV

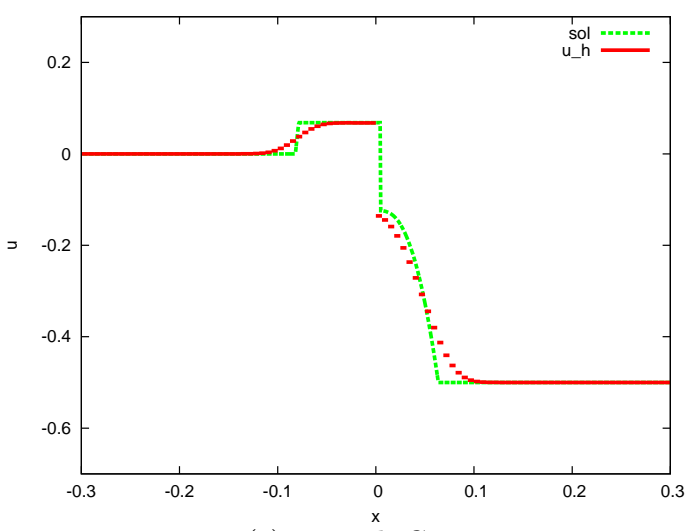

(c) RR-sub-GE

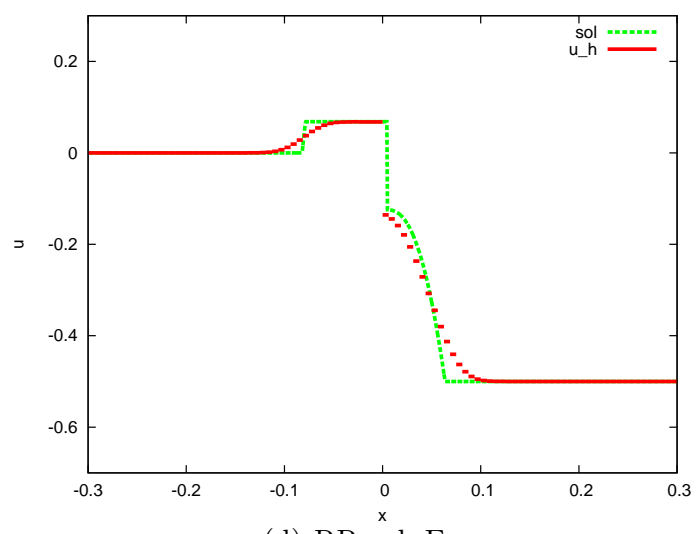

(d) RR-sub-E

Figure 5: Comparing different Riemann solvers. Parameters are chosen as $\Delta \xi=0.2, \varepsilon=10^{-3}$ and $N=50$. The numerical solution is plotted in red, the exact in green (dashed line). We skipped the $\tau$-plot for RRsub-GE and RR-sub-E solvers, since there is no visible difference to the RR-sub-FV solver. We used a mesh with 320 cells. 


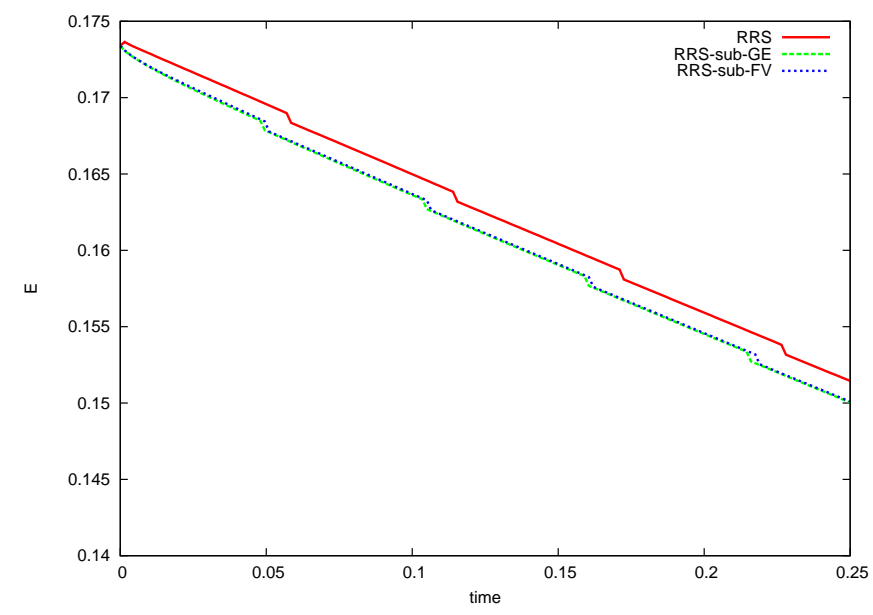

Figure 6: Decay of total energy $E_{h}$ for overall scheme with RR (red), RR-sub-GE (green) and RR-sub-FV (blue) solvers.

\subsubsection{Tracking Algorithm 2.1 with Approximate Riemann Solvers Us- ing a Non-linear Kinetic Relation}

We demonstrate, how the new Riemann solver works with non-linear kinetic relations. We consider the kinetic relation (3.12) given in Example 3.2 from Section 3.1.

Figure 9 displays the results for a Riemann problem. The initial conditions are chosen as in (3.35). If we compare the results with those displayed in Figure 5, then we can observe a similar behavior. We indeed have a "non-monotonic" behavior for the approximate Riemann solver and a "monotonic" one for the others. Note that for this nonlinear kinetic relation an exact Riemann solver is not available.

\section{Relaxation Riemann Solvers for Thermoelastic Ma- terials}

\subsection{The Mathematical Model}

We extend the barotropic system (3.1) to the thermoelastic situation with the special free energy

$$
\Psi=\Psi(\tau, \theta)=\frac{1}{4} \tau^{4}-\frac{1}{2} b \tau^{2}\left(\theta-\theta_{0}\right)-c \theta \log \left(\frac{\theta}{\theta_{0}}\right),
$$

where $\theta>0$ is the temperature. Next to the constants $c,-b>0$ we denote by $\theta_{0}>0$ the critical temperature. We consider the subcritical situation $\theta<\theta_{0}$ such that the free energy $\Psi(\cdot, \theta)$ has double-well structure, being convex for

$$
\tau \in \mathcal{Z}:=\mathcal{Z}_{1} \cup \mathcal{Z}_{2}:=\left(-\infty,-\sqrt{\frac{b\left(\theta-\theta_{0}\right)}{3}}\right) \cup\left(\sqrt{\frac{b\left(\theta-\theta_{0}\right)}{3}}, \infty\right) .
$$

With (4.1) the specific entropy $\eta=\eta(\tau, \theta)$ and the pressure $p=p(\tau, \theta)$ are given through

$$
\begin{aligned}
& \eta(\tau, \theta)=-\Psi_{\theta}(\tau, \theta), \\
& p(\tau, \theta)=-\Psi_{\tau}(\tau, \theta) .
\end{aligned}
$$




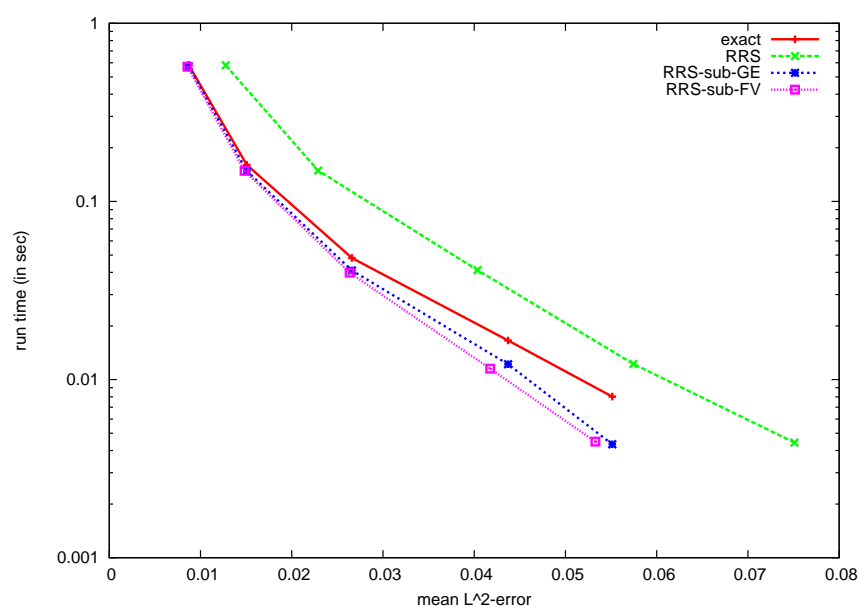

Figure 7: Runtime-error comparison for overall scheme with RR (green), RR-sub-GE (blue), RR-sub-FV (pink)solvers and an exact Riemann solver (red).

With the internal energy $e=\Psi+\eta \theta$ and the total energy $E=e+u^{2} / 2$ a thermoelastic process for unknown $U=(u, \tau, E)^{T}$ is governed by the equations

$$
\begin{aligned}
& \partial_{t} u+\partial_{x} p(\tau, \theta)=0, \\
& \partial_{t} \tau-\partial_{x} u=0, \\
& \partial_{t} E+\partial_{x}(p(\tau, \theta) u)=0 .
\end{aligned}
$$

It is easy to verify that (4.3) is strictly hyperbolic in

$$
U_{1 / 2}=\left\{(u, \tau, E)^{T} \mid u \in \mathbb{R}, \tau \in \mathcal{Z}_{1 / 2}, \theta \in\left(0, \theta_{0}\right)\right\},
$$

where we consider $\theta$ as a function of $U$ :

$$
\theta(u, \tau, E)=\frac{1}{c}\left(E-\frac{u^{2}}{2}-\frac{1}{4} \tau^{4}-\frac{1}{2} b \tau^{2} \theta_{0}\right) .
$$

The solution of a Riemann problem for (4.3) with data in different phases is expected to have a wave-structure as shown in Figure 10 for positive phase boundary speed.

To ensure uniqueness a kinetic relation has to be supplemented. We consider the (temperature-independent) relation from Example 3.1. A non-linear and temperature dependent example (cf. Abeyaratne\&Knowles [3]) is provided by (3.11) if (3.10) is now defined by

$$
f=\Psi_{+}-\Psi_{-}+\frac{1}{2}\left(p_{+}+p_{-}\right)\left(\tau_{+}-\tau_{-}\right)+\frac{1}{2}\left(\eta_{+}+\eta_{-}\right)\left(\theta_{+}-\theta_{-}\right) .
$$

\subsection{Relaxation Approximation and Sub-Iteration}

The relaxation approximation (3.13) for thermoelastic materials we consider hereafter is described in [9] in the case of purely hyperbolic models. It closely follows the strategy proposed in the barotropic setting considering a relaxation pressure $\Pi$ governed by its own PDE with stiff relaxation. We also refer for a complementary presentation to [5, 8]. Extension of the proposed relaxation procedure to the framework of materials with phase transition is again motivated by the wave structure of non-classical Riemann solutions displayed in Figure 10. One additional wave accounting for phase transition is indeed 


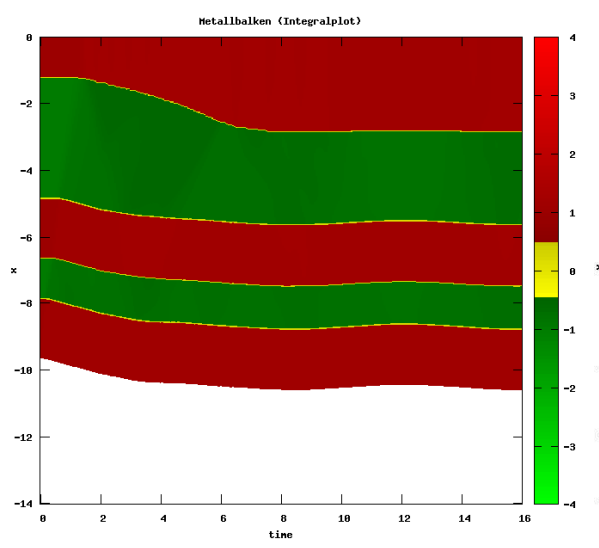

(a) $\kappa=\frac{1}{2}$, low weight.

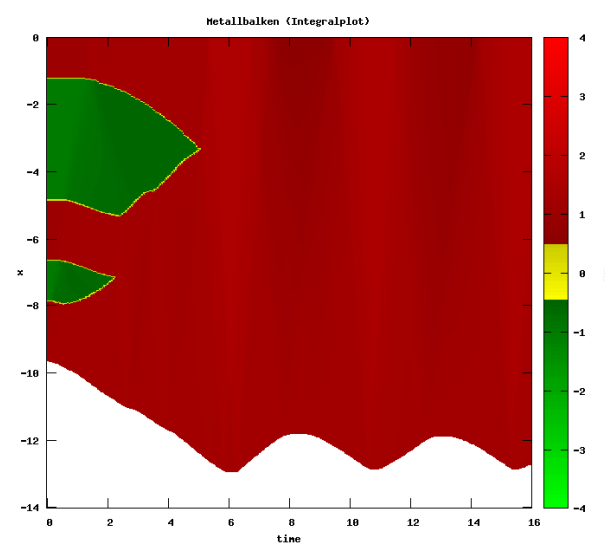

(c) $\kappa=\frac{1}{2}$, heavy weight.

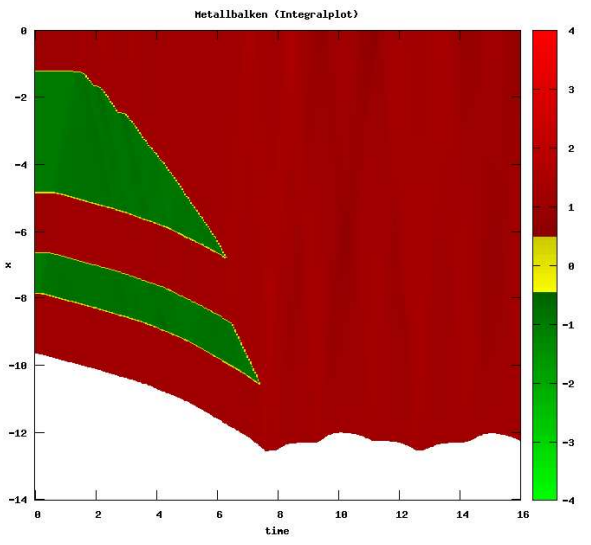

(b) $\kappa=\frac{9}{10}$, low weight.

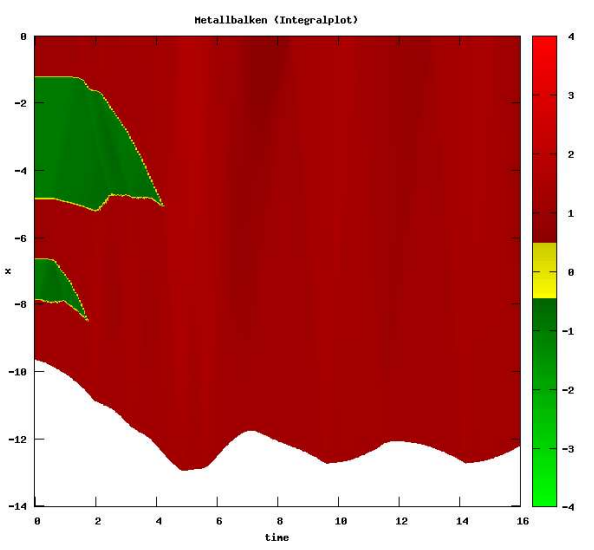

(d) $\kappa=\frac{9}{10}$, heavy weight.

Figure 8: Simulation of a hanging elastic bar (vertical axis corresponds to space, horizontal axis to time!) with different kinetic relations. The colors scale with the stress value.

introduced when required, considering the following PDE system with singular perturbation:

$$
\begin{aligned}
& \partial_{t} u+\partial_{x} \Pi=0, \\
& \partial_{t} \tau-\partial_{x} u=0 \text {, } \\
& \partial_{t} E+\partial_{x} \Pi u=\mathcal{M}\left(U_{L}, U_{R}\right) \delta_{x-\sigma\left(U_{L}, U_{R}\right) t} \text {, } \\
& \partial_{t} \Pi+a^{2} \partial_{x} u=0 \text {. }
\end{aligned}
$$

A Dirac measure is concentrated on an approximate transition wave propagating with speed $\sigma\left(U_{L}, U_{R}\right)$ given by

$$
\sigma\left(U_{L}, U_{R}\right)=-\frac{u_{R}-u_{L}}{\tau_{R}-\tau_{L}}
$$

while the corresponding mass $\mathcal{M}\left(U_{L}, U_{R}\right)$ is prescribed so as to fulfill the kinetic relation. The solution of the Riemann problem for (4.5) has the wave structure shown in Figure 11. The jump conditions (3.21), (3.22) and (3.23) introduced in the barotropic setting in Section 3.2 are again obviously in order. Then and concerning the jump conditions for governing the intermediate total energies, it can be easily checked from (3.21) that the 

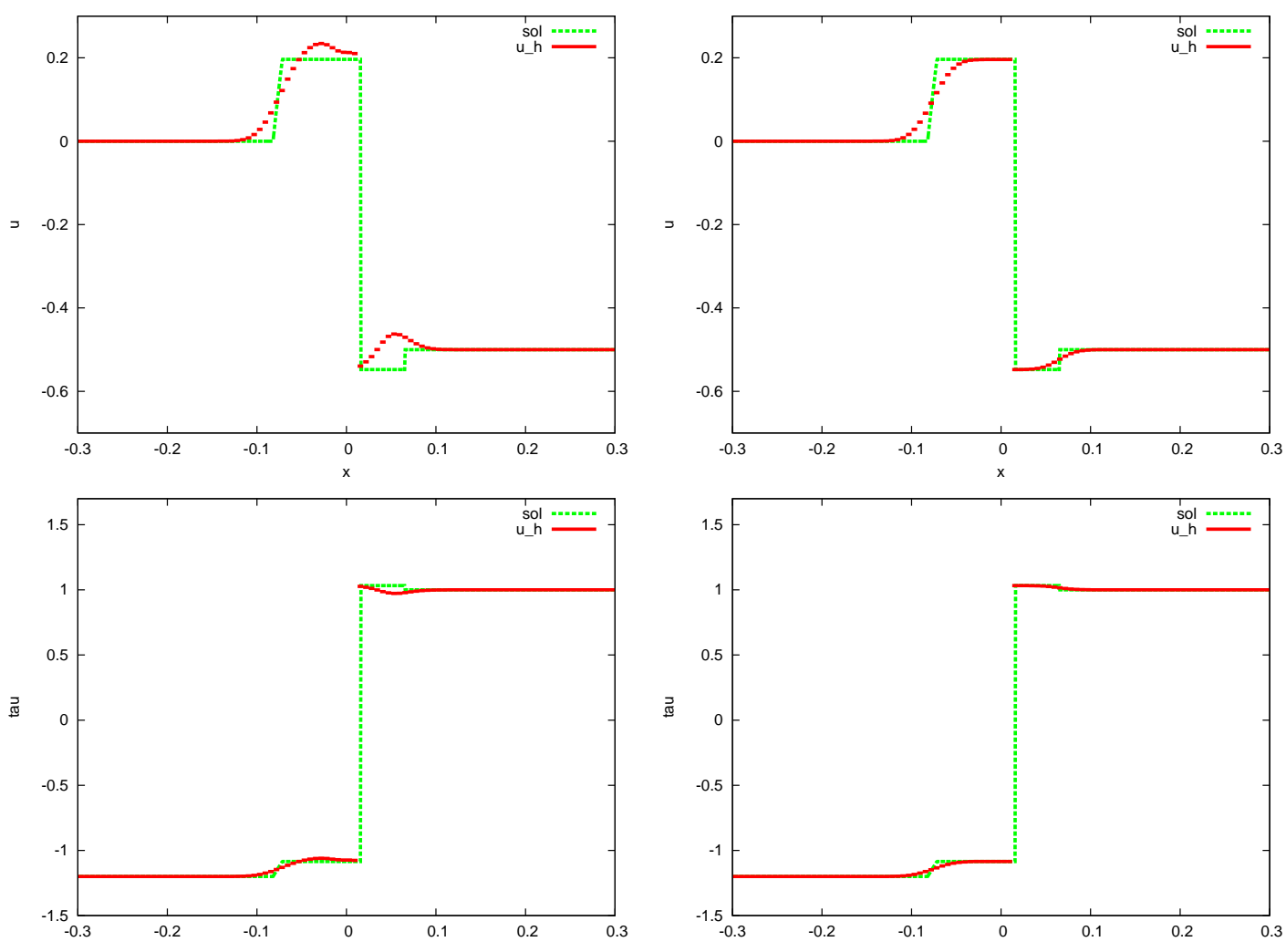

(a) $\mathrm{RR}$

(b) RR-sub-FV (RR-sub-GE and RR-sub-E)

Figure 9: Comparing different approximate Riemann solvers for a nonlinear kinetic relation. Parameters are chosen as $\Delta \xi=0.2, \varepsilon=10^{-3}$ and $N=50$. The numerical solution is plotted in red (solid line), the exact in green (dashed line). We skipped the plots for RR-sub-GE and RR-sub-E methods, since there is no visible difference to the RR-sub-FV version. We used a grid with 320 cells.

two extreme waves obey the following jump relations:

$$
\pm a\left[\frac{u^{2}}{2}+\frac{\Pi}{2 a^{2}}\right]+[\Pi u]=0
$$

together with

$$
\pm a[E]+[\Pi u]=0
$$

We thus readily infer the following formulae

$$
\begin{gathered}
E_{\#}^{*}-\frac{1}{2} u_{\#}^{* 2}-\frac{1}{2 a^{2}} \Pi_{\#}^{* 2}=E_{L}-\frac{1}{2} u_{L}^{2}-\frac{1}{2 a^{2}} \Pi_{L}^{2}, \\
E_{R}-\frac{1}{2} u_{R}^{2}-\frac{1}{2 a^{2}} \Pi_{R}^{2}=E_{+}^{*}-\frac{1}{2} u_{+}^{* 2}-\frac{1}{2 a^{2}} \Pi_{+}^{* 2} .
\end{gathered}
$$

At the approximate transition wave, we easily get :

$$
\sigma\left(E_{+}^{*}-E_{-}^{*}\right)=\Pi_{+}^{*} u_{+}^{*}-\Pi_{-}^{*} u_{-}^{*} .
$$

If we are in the case of a kinetic relation, which is independent of the temperature, then we are finished by now since the jump conditions for the total energy stay completely 


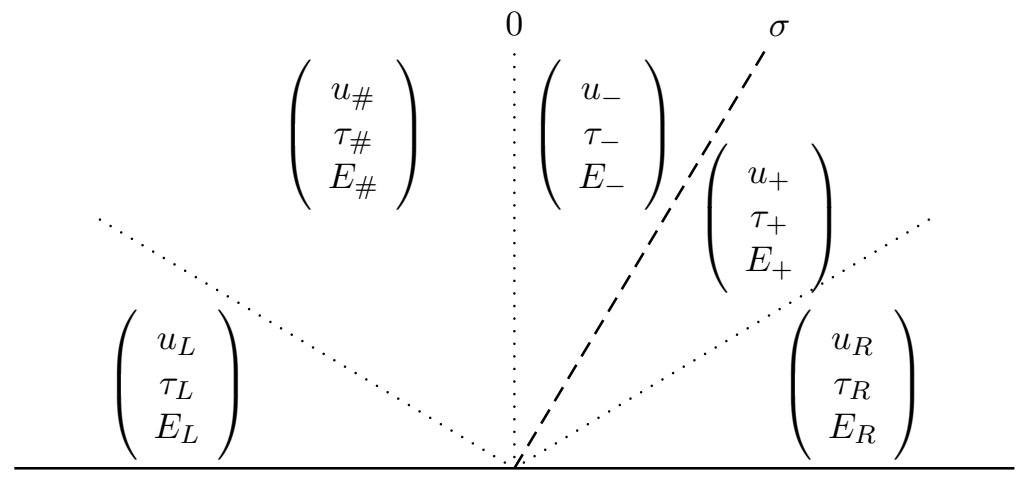

Figure 10: Wave structure of a Riemann solution of (4.3)for tho-phase initial datum.

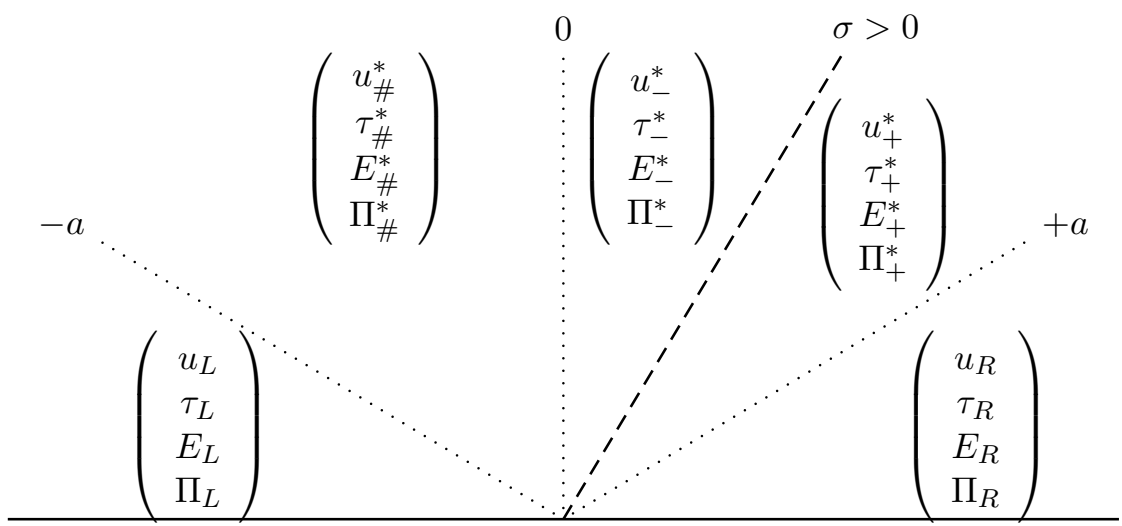

Figure 11: Wave structure of an approximate Riemann solution of (4.3) for two-phase initial datum.

from the others. In other words, the formulae (3.17), Section 3.2, for defining $u_{-}^{*}, \tau_{-}^{*}, \Pi_{-}^{*}$, $u_{+}^{*}, \tau_{+}^{*}$ and $\Pi_{+}^{*}$ are kept unchanged. It then suffices to plug these formulae in (4.6) and (4.7) to calculate $E_{-}^{*}$ and $E_{+}^{*}$.

In the remaining section we have a closer look at kinetic relations, which depend on $E_{-}$ and $E_{+}($as $(3.11)$ with (4.4)). In this case we first solve the linear equations (3.21), (3.22) and (3.23) to get a one-dimensional solution space, which we can linearly parameterize by a variable $s$. By (4.6) and (4.7) we get quadratic polynomial formulas for $E_{-}^{*}=E_{-}^{*}(s)$ and $E_{+}^{*}=E_{+}^{*}(s)$. All together it remains to solve the kinetic relation, which has the form

$$
\mathcal{K}\left(U_{-}^{*}(s), U_{+}^{*}(s)\right)=0
$$

where $U_{ \pm}^{*}=\left(u_{ \pm}^{*}, \tau_{ \pm}^{*}, E_{ \pm}^{*}\right)^{T}$. Even if the kinetic relation $\mathcal{K}$ is linear, we have to solve a quadratic equation. From computations with the above-mentioned example it seems to be that choosing $a$ big enough ensures the unique solvability in the hyperbolic phase space.

For the sake of improvement the relaxation solver can be used in combination with sub-iterative methods as introduced in Section 3.3. However let us point out that only the versatile RR-sub-FV version is applicable as the RR-sub-GE/RR-sub-E methods rely on special properties of the barotropic system. 


\subsection{Numerical Experiments}

For thermoelastic materials with the proposed kinetic relations we are not aware of complete exact Riemann solutions. Therefore this section is not devoted to the analysis of our numerical approach but serves merely as way to gain more understanding on the thermoelastic Riemann problem.

Let us have a look at the Riemann problem with initial conditions
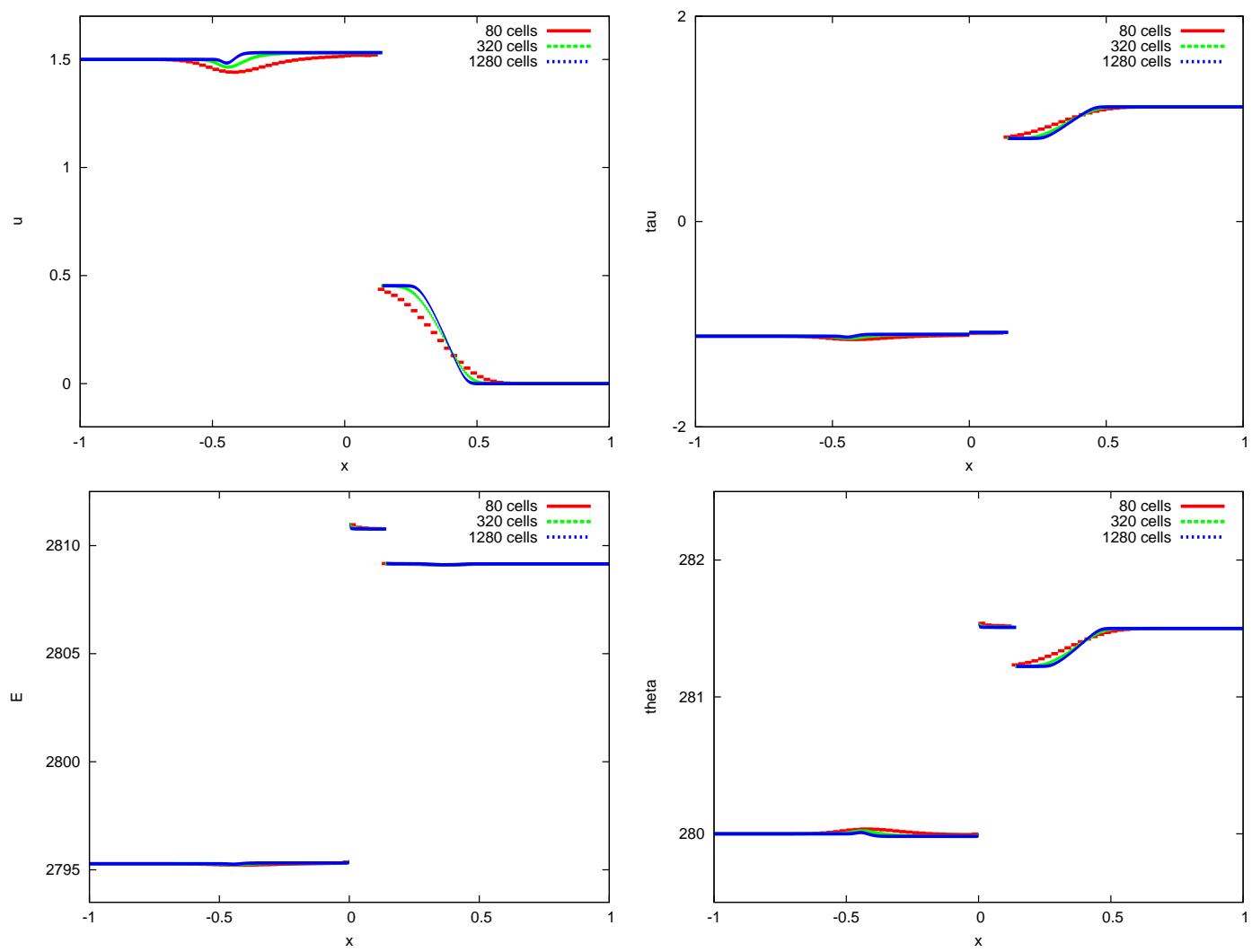

Figure 12: Riemann problem (4.9) solved with RR solver for three equidistant grids with 80,320 and 1280 volumes.

$$
U_{L}=\left(\begin{array}{c}
1.5 \\
-\tau_{M}-0.3 \\
E_{L}
\end{array}\right) \quad \text { and } \quad U_{R}=\left(\begin{array}{c}
0.0 \\
\tau_{M}+0.3 \\
E_{R}
\end{array}\right)
$$

The state $\tau_{M}>0$ is a Maxwell-state given by $\tau_{M}=\sqrt{b\left(\theta-\theta_{0}\right)}$. The total energies $E_{L / R}$ are chosen such that such that $\theta_{L}=\theta_{*}$ and $\theta_{R}=\theta_{*}+1.5$ holds. The parameters are

$$
\theta_{*}=280.0, \quad \theta_{0}=300.0, \quad c=10.0 \quad \text { and } \quad b=-\frac{1}{30} .
$$

In the first example we use the kinetic relation

$$
\tau_{+}=-\kappa \tau_{-} \quad \text { with } \kappa=\frac{3}{4} .
$$

The overall scheme converges to the same function for the RR as well as the RR-sub-FV solver, see Figures 12 and 13. Figure 12 shows the over-all schemes for different grids. 

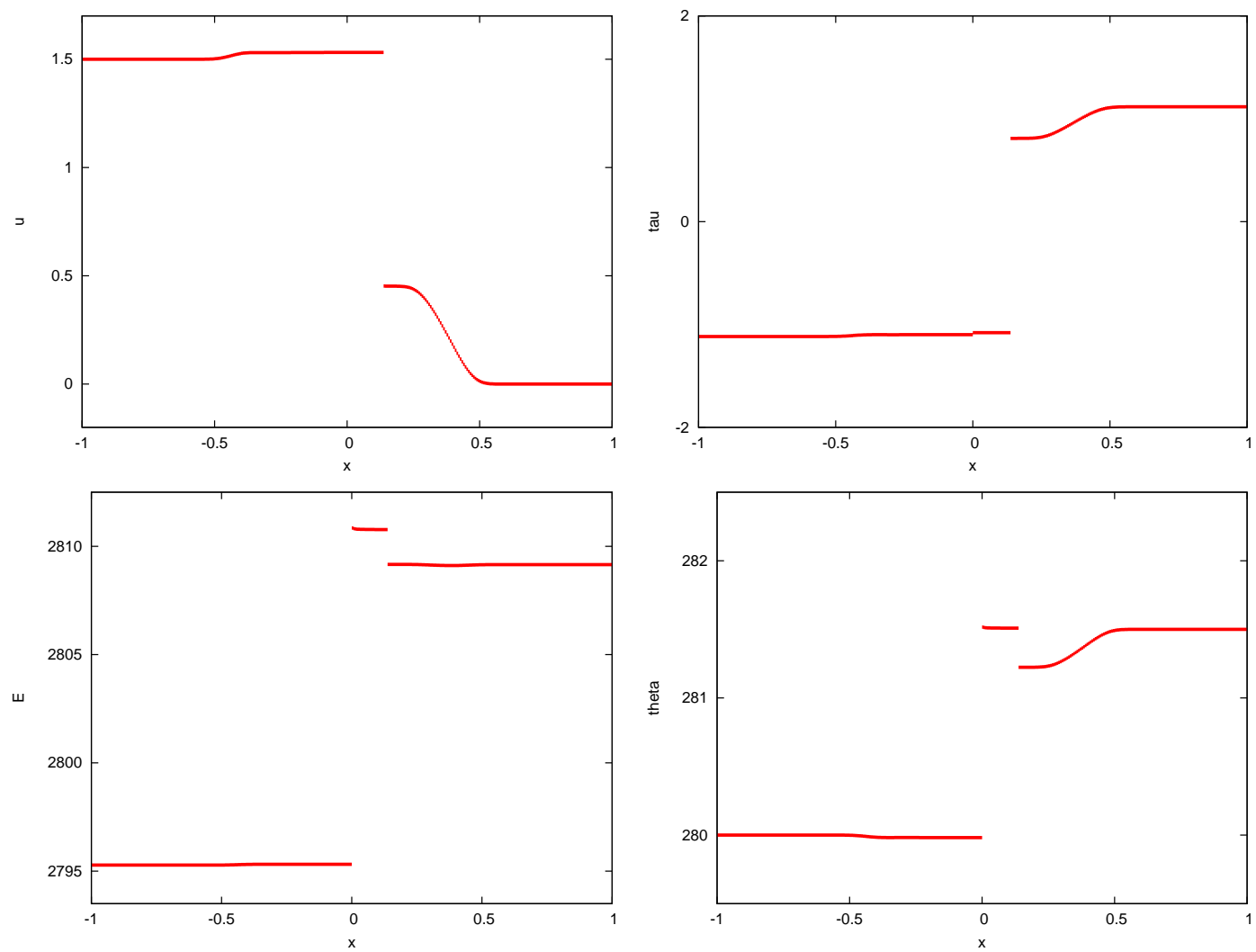

Figure 13: Riemann problem (4.9) solved with RR-sub-FV solver for a equidistant grid with 320 volumes.

As in the barotropic case we observe over/undershoots for the RR solver, while the RRsub-FV solver behaves monotonically. As expected we observe a solution made up of four waves. Note that the phase boundary and the contact discontinuity are sharply captured. The latter property is due to the fact that we use a relaxation numerical flux in the bulk phase which exactly captures contact discontinuities at rest.

In the next example we have constructed an exact solution of a Riemann problem, involving only two waves. It consists of a shock connecting $U_{L}$ to $U_{M}$ and a phase boundary connecting $U_{M}$ to $U_{R}$. The exact values are

$$
U_{L}=\left(\begin{array}{c}
u_{L} \\
\tau_{L} \\
E_{L}
\end{array}\right)=\left(\begin{array}{c}
\frac{\sqrt{8737338}}{1202} \\
-1 \\
\frac{322625}{1202}
\end{array}\right), \quad U_{M}=\left(\begin{array}{c}
u_{M} \\
\tau_{M} \\
E_{M}
\end{array}\right)=\left(\begin{array}{c}
0 \\
-2 \\
2684
\end{array}\right)
$$

and

$U_{R}=\left(\begin{array}{c}u_{R} \\ \tau_{R} \\ E_{R}\end{array}\right)=\left(\begin{array}{c}\left(-\frac{7}{18212988}+\frac{1}{54638964} \sqrt{3}\right) \sqrt{190106437188982-13135845917929 \sqrt{3}} \\ \frac{1}{6}(9-\sqrt{3}) \\ \frac{877339726147}{327833784}+\frac{33039211}{9106494} \sqrt{3}\end{array}\right)$.

The results are plotted In Figure 14. In the numerical solution there are relicts of the contact discontinuity and the 3 -wave (the so-called wall-heating phenomenon [24]). Nevertheless the method seems to converge to the correct solution as already observed in the classical Lagrangian setting. 

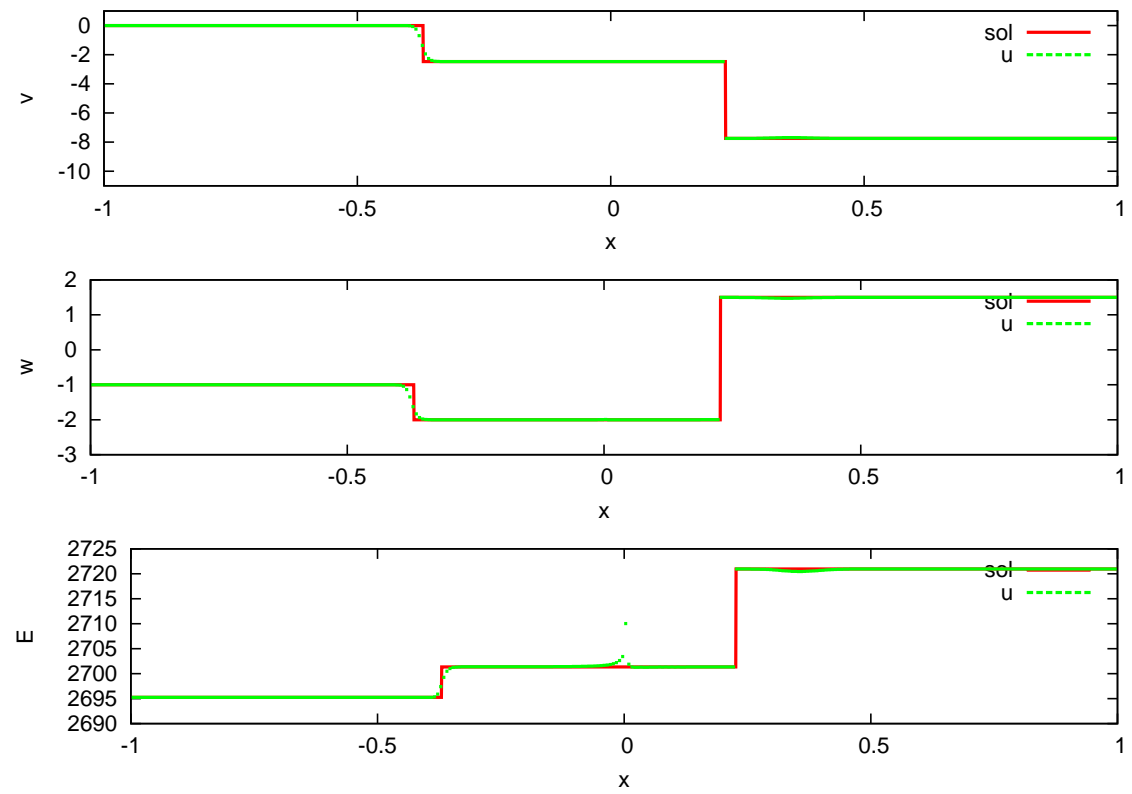

Figure 14: Initial value problem solved with RR-sub-FV (green) with reference solution (red).

\section{References}

[1] R. Abeyaratne and J. K. Knowles. Kinetic relations and the propagation of phase boundaries in solids. Arch. Rational Mech. Anal., 114(2):119-154, 1991.

[2] R. Abeyaratne and J. K. Knowles. A continuum model of a thermoelastic solid capable of undergoing phase transitions. J. Mech. Phys. Solids, 41(3):541-571, 1993.

[3] R. Abeyaratne and J. K. Knowles. Evolution of phase transitions, a continuum theory. Cambridge University Press, 2006.

[4] A. Berezovski and G. A. Maugin. Numerical simulation of phase-transition front propagation in thermoelastic solids. In Numerical mathematics and advanced applications, pages 703-711. Springer, Berlin, 2006.

[5] F. Bouchut. Nonlinear stability of finite volume methods for hyperbolic conservation laws and well-balanced schemes for sources. Frontiers in Mathematics. Birkhäuser Verlag, Basel, 2004.

[6] B. Boutin, C. Chalons, F. Lagoutière, and P. G. LeFloch. Convergent and conservative schemes for nonclassical solutions based on kinetic relations. I. Interfaces Free Bound., 10(3):399-421, 2008.

[7] C. Chalons. Transport-equilibrium schemes for computing nonclassical shocks. Scalar conservation laws. Numer. Methods Partial Differential Equations, 24(4):1127-1147, 2008.

[8] C. Chalons and F. Coquel. Navier-Stokes equations with several independent pressure laws and explicit predictor-corrector schemes. Numer. Math., 101(3):451-478, 2005.

[9] C. Chalons and J.-F. Coulombel. Relaxation approximation of the Euler equations. J. Math. Anal. Appl., 348(2):872-893, 2008.

[10] C. Chalons and P. Goatin. Transport-equilibrium schemes for computing contact discontinuities in traffic flow modeling. Commun. Math. Sci., 5(3):533-551, 2007. 
[11] C. Chalons and P. Goatin. Godunov scheme and sampling technique for computing phase transitions in traffic flow modeling. Interfaces Free Bound., 10(2):197-221, 2008.

[12] C. Chalons and P. G. LeFloch. Computing undercompressive waves with the random choice scheme. Nonclassical shock waves. Interfaces Free Bound., 5(2):129-158, 2003.

[13] F. Coquel and B. Perthame. Relaxation of energy and approximate Riemann solvers for general pressure laws in fluid dynamics. SIAM J. Numer. Anal., 35(6):2223-2249, 1998.

[14] A. Corli and H. Fan. The Riemann problem for reversible reactive flows with metastability. SIAM J. Appl. Math., 65(2):426-457, 2004/05.

[15] A. Dressel and C. Rohde. A finite-volume approach to liquid-vapour fluids with phase transition. In Finite volumes for complex applications $V$, pages 53-68. ISTE, London, 2008.

[16] H. Hattori. The Riemann problem for thermoelastic materials with phase change. $J$. Differential Equations, 205(1):229-252, 2004.

[17] S. Jin and Z. P. Xin. The relaxation schemes for systems of conservation laws in arbitrary space dimensions. Comm. Pure Appl. Math., 48(3):235-276, 1995.

[18] P. G. LeFloch. Hyperbolic Systems of Conservation Laws. The theory of classical and nonclassical shock waves. Lectures in Mathematics ETH Zürich. Birkhäuser Verlag, Basel, 2002.

[19] P. G. LeFloch, J. M. Mercier, and C. Rohde. Fully discrete, entropy conservative schemes of arbitrary order. SIAM J. Numer. Anal., 40(5):1968-1992, 2002.

[20] P. G. LeFloch and M. D. Thanh. Non-classical Riemann solvers and kinetic relations. II. An hyperbolic-elliptic model of phase-transition dynamics. Proc. Roy. Soc. Edinburgh Sect. A, 132(1):181-219, 2002.

[21] J.-M. Mercier and B. Piccoli. Admissible Riemann solvers for genuinely nonlinear p-systems of mixed type. J. Differential Equations, 180(2):395-426, 2002.

[22] C. Merkle and C. Rohde. Computation of dynamical phase transitions in solids. Appl. Numer. Math., 56(10-11):1450-1463, 2006.

[23] C. Merkle and C. Rohde. The sharp-interface approach for fluids with phase change: Riemann problems and ghost fluid techniques. M2AN Math. Model. Numer. Anal., 41(6):1089-1123, 2007.

[24] W. Noh. Errors for calculations of strong shocks using an artificial viscosity and an artificial heat flux. Journal of Computational Physics, 72(1):78 - 120, 1987.

[25] V. Perrier. A conservative method for the simulation of the isothermal euler system with the van-der-waals equation of state. Journal of Scientific Computing, 48:296303, 2011. 10.1007/s10915-010-9415-9.

[26] I. Suliciu. On the thermodynamics of rate-type fluids and phase transitions. I. Ratetype fluids. International Journal of Engineering Science, 36(9):921 - 947, 1998.

[27] L. Truskinovsky. Kinks versus shocks. In Shock induced transitions and phase structures in general media, volume 52 of IMA Vol. Math. Appl., pages 185-229. Springer, New York, 1993.

[28] X. Zhong, T. Y. Hou, and P. G. LeFloch. Computational methods for propagating phase boundaries. J. Comput. Phys., 124(1):192-216, 1996. 